\title{
Artificial neural networks: a practical review of applications involving fractional calculus
}

\author{
E. Viera-Martin ${ }^{1}$, J. F. Gómez-Aguilar ${ }^{2, a}{ }_{\mathbb{C}}$, J. E. Solís-Pérez ${ }^{3}$, J. A. Hernández-Pérez ${ }^{4}$, and \\ R. F. Escobar-Jiménez ${ }^{1}$ \\ 1 Tecnológico Nacional de México/CENIDET, Interior Internado Palmira S/N, Col. Palmira, C.P. 62490 Cuernavaca, \\ Morelos, Mexico \\ 2 CONACyT-Tecnológico Nacional de México/CENIDET, Interior Internado Palmira S/N, Col. Palmira, C.P. 62490 \\ Cuernavaca, Morelos, Mexico \\ 3 Escuela Nacional de Estudios Superiores Unidad Juriquilla, Universidad Nacional Autónoma de México, Boulevard \\ Juriquilla 3001, Juriquilla La Mesa, C.P. 76230 Juriquilla, Querétaro, Mexico \\ 4 Universidad Autónoma del Estado de Morelos/Centro de Investigación en Ingeniería y Ciencias Aplicadas, Av. Universidad \\ No. 1001, Col Chamilpa, C.P. 62209 Cuernavaca, Morelos, Mexico
}

Received 7 March 2021 / Accepted 13 January 2022 / Published online 12 February 2022

(C) The Author(s), under exclusive licence to EDP Sciences, Springer-Verlag GmbH Germany, part of Springer Nature 2022

\begin{abstract}
In this work, a bibliographic analysis on artificial neural networks (ANNs) using fractional calculus (FC) theory has been developed to summarize the main features and applications of the ANNs. ANN is a mathematical modeling tool used in several sciences and engineering fields. FC has been mainly applied on ANNs with three different objectives, such as systems stabilization, systems synchronization, and parameters training, using optimization algorithms. FC and some control strategies have been satisfactorily employed to attain the synchronization and stabilization of ANNs. To show this fact, in this manuscript are summarized, the architecture of the systems, the control strategies, and the fractional derivatives used in each research work, also, the achieved goals are presented. Regarding the parameters training using optimization algorithms issue, in this manuscript, the systems types, the fractional derivatives involved, and the optimization algorithm employed to train the ANN parameters are also presented. In most of the works found in the literature where ANNs and FC are involved, the authors focused on controlling the systems using synchronization and stabilization. Furthermore, recent applications of ANNs with FC in several fields such as medicine, cryptographic, image processing, robotic are reviewed in detail in this manuscript. Works with applications, such as chaos analysis, functions approximation, heat transfer process, periodicity, and dissipativity, also were included. Almost to the end of the paper, several future research topics arising on ANNs involved with FC are recommended to the researchers community. From the bibliographic review, we concluded that the Caputo derivative is the most utilized derivative for solving problems with ANNs because its initial values take the same form as the differential equations of integer-order.
\end{abstract}

\section{Introduction}

Artificial neural networks (ANNs) have emerged as a promising alternative to simulate systems due to their successful applications in several engineering and science fields, such as signal processing, image processing, control systems, associative memory, to name a few. Besides, fractional calculus (FC) is an extension and generalization of the integer-order calculus, which its main characteristic is the memory description. When the ANNs are modeled using fractional differential equations (FDE), they are named fractional artificial neural networks (FANNs). The FDE is used

\footnotetext{
a e-mail: jose.ga@cenidet.tecnm.mx (corresponding author)
}

for describing the dynamical behavior of the ANNs neurons. Hence, in the last decade, many authors employed FANN for modeling physics and engineering systems more efficiently and accurately.

In this manuscript, we will use the term "ANN involved with FC" to refer to the FANN. Compared with the ordinary ANNs, ANNs involved with FC have important advantages, such as the description of memory and hereditary properties of several processes; and the system performance is enriched due to one more degree of freedom [1]. Fractional-order systems can process information efficiently, improving the simulations of the integer-order systems, finding more accurate results. Since many of the real-world problems can be generally identified and described by the fractional- 
order models [2], it can be expected the same or better results could be reached using the FANN.

The key aspects of this review are the study and comprehension of the main derivatives related to FC. Several definitions of fractional derivatives have been proposed, for example, Grünwald-Letnikov, RiemannLiouville, Caputo, Caputo-Fabrizio, and AtanganaBaleanu fractional derivatives. The Riemann-Liouville and Caputo derivatives use the power-law kernel. The Caputo-Fabrizio derivative uses an exponential kernel, and the Atangana-Baleanu derivative utilizes the generalized Mittag-Leffler function as the nonsingular and nonlocal kernel. The equations described by fractional derivatives are highly complex, and there exist lots of analytical and numerical methods to solve them. These techniques have allowed establishing a comparison point between the exact solution and the approximation carried out by the ANN involved with FC. Several numerical or analytical methods that have been developed to solve FDE are, for instance: the Adams-Bashfort-Moulton method, homotopy perturbation method, variational iteration method, Adomian decomposition method, Laplace transform method, among others [3-10].

The uncertainty of parameters affects the modeling and controlling of the systems we are dealing with. Therefore, parameter estimation of ANN involved with FC is crucial for the theoretical study and practical applications [11]. In training ANN involved with FC, the synaptic connection weights between different neurons are adjusted. The weights training is carried out using optimization algorithms. An optimization algorithm is an efficient searching method to solve constrained optimization problems [12]. The various optimization algorithms to train ANN involved with FC include the algorithms based on back-propagation (BP), such as gradient descent algorithm (GD) or Levenberg-Marquardt algorithm (LM) among others, and algorithms based on heuristic methods, such as genetic algorithm (GA), simulating annealing algorithm (SA), particle swarm optimization algorithm (PSO), and so on. At present, synchronization of chaotic fractional-order differential systems becomes a challenging and interesting problem due to its potential applications and the ability to model systems accurately. Amongst all kinds of fractional-order chaos synchronization, the most commonly employed to synchronize ANN involved with FC are projective synchronization, global synchronization, finite-time synchronization, quasi synchronization, and adaptive synchronization. Nowadays, many authors have published works where sufficient conditions are derived, to achieve these types of synchronizations on ANN involved with FC $[13,14]$. Many control techniques have been used to show their synchronization, among them: feedback control, adaptive control, sliding mode control, impulsive control, and so on [15]. Moreover, the stability analysis of ANN is one of the most important and active areas of research. Consequently, some improved stabilization methods for different types of nonlinear systems are worthy of further investigation [16]. Stability analysis of several systems has been investigated since they have been successfully applied in some engineering fields, such as signal processing, pattern classification, control, and optimization [14]. In recent years, FC is introduced for the stability analysis of nonlinear systems, allowing us to study the most important stability types, such as exponential stability, finite-time stability, uniform stability, global stability, etc. Several control techniques have been widely used to guarantee stability on ANN involved with FC, among them, sliding mode control, feedback control, and impulsive control [13,14].

Besides, the ANN involved with $\mathrm{FC}$ has been employed in approximation, estimation, control of chaos. Moreover, it has been found an ANN involved with FC with applications in cryptographic, medicine, sustainable energy, images, circuit realization, unmanned aerial vehicles, and robotics.

In the present work, a state of the art review related to the ANN involved with FC is carried out. This paper is organized as follows: Sect. 2 presents a synthesis about the FC applied to ANN; in Sect. 3, the analytical and numerical methods employed to solve the Differential Equations (DE) and FDE that model the concerned systems are reviewed. Subsequently, Sect. 4 presents a thorough overview of the optimization algorithms employed for the training of ANN involved with FC; in Sect. 5, the control strategies employed to synchronize and stabilize ANN involved with FC are summarized. Section 6 shows other important applications of ANN involved with FC. In Sect. 7, some future directions about ANN involved with FC are given. Finally, Sect. 8 presents a summary of the most relevant information of this research.

\section{Mathematical preliminaries}

In this section, we present some fractional-order derivatives widely used in the fractional calculus.

Definition 1 Let $\alpha \in \mathbb{R}_{+}$and $n=\lceil\alpha\rceil$. The fractional operator in the Riemann-Liouville sense is given as follows [17]

$$
\begin{aligned}
& { }_{0}^{R L} \mathscr{D}_{t}^{\alpha}\{f(t)\}=\frac{1}{\Gamma(n-\alpha)} \frac{\mathrm{d}^{n}}{\mathrm{~d} t^{n}} \\
& \int_{a}^{t} \frac{f(\tau)}{(t-\tau)^{\alpha-n+1}} \mathrm{~d} \tau, \quad n-1<\alpha \leq n,
\end{aligned}
$$

where $a$ and $t$ are the limits of operation of ${ }_{0}^{R L} \mathscr{D}_{t}^{\alpha}\{f(t)\}$ and $\Gamma(\cdot)$ is the Euler Gamma function.

Definition 2 Let $\alpha \in \mathbb{R}_{+}$. The fractional operator of Grünwald-Letnikov is given by [17]

$$
G L \mathscr{D}_{t}^{\alpha}\{f(t)\}=\lim _{h \rightarrow 0} \frac{\left(\triangle_{h}^{\alpha} f\right)(x)}{h^{\alpha}},
$$

where, 


$$
\left(\triangle_{h}^{\alpha} f\right)(x)=\sum_{k=0}^{\infty}(-1)^{k}\left(\begin{array}{l}
\alpha \\
k
\end{array}\right) f(x-k h), \quad \alpha>0,
$$

and $\left(\begin{array}{l}\alpha \\ k\end{array}\right)$ is the generalized binomial coefficient.

Definition 3 The Liouville-Caputo fractional derivative, which will be named as Caputo derivative in the rest of this paper, is defined as the convolution of the local derivative of a given function with a power-law kernel. Therefore, the derivative of order $(\alpha>0)$ is defined as follows [17]

$$
\begin{aligned}
& { }_{0}^{C} \mathscr{D}_{t}^{\alpha}\{f(t)\}=\frac{1}{\Gamma(n-\alpha)} \\
& \quad \times \int_{0}^{t} \frac{f^{(n)}(\tau)}{(t-\tau)^{\alpha-n+1}} \mathrm{~d} \tau, \quad n-1<\alpha \leq n .
\end{aligned}
$$

Definition 4 Let $f \in H^{1}(a, b), b>a, \alpha \in[0,1)$ then, the Atangana-Baleanu fractional derivative in Liouville-Caputo sense is given as [18]

$$
\begin{aligned}
& \underset{0}{A B C} \mathscr{D}_{t}^{\alpha}\{f(t)\}=\frac{A B(\alpha)}{n-\alpha} \\
& \quad \times \int_{0}^{t} f^{(n)}(\tau) E_{\alpha}\left[-\alpha \frac{(t-\tau)^{\alpha}}{n-\alpha}\right] \mathrm{d} \tau, \quad n-1<\alpha<n,
\end{aligned}
$$

where $A B(\alpha)$ is a normalization function.

\section{Methods to solve fractional differential equations}

There are different analytical and numerical methods to solve DE and FDE. In this section are introduced the methods used to solve the DE and FDE on ANN involved with FC. Also, in this section, a brief discussion is carried out to summarize some of these methods. In [19], a chaotic memristive Hopfield FANN was designed using the 4th order Runge-Kutta numerical method involving the Grünwald-Letnikov derivative. The dynamical properties of the system were studied. And an adaptive sliding mode control was used for the system synchronization. In Ref. [20], the problems on the stability and synchronization of quaternion-valued FANN was investigated involving Caputo derivative, and the Adams-Bashforth-Moulton predictor-corrector method was used to solve the FDE and demonstrate the validity of the theoretical results. On the other hand, in [21], an orthogonal FANN was employed to solve various types of Lane-Emden equations that arise in several physical phenomena. The fractional-order Lane-Emden equation was generalized by considering its derivative in Caputo sense, the analytical approximation to the solution of the FDE was carried out using Adomian decomposition method. In Ref. [22], an orthogonal Jacobi FANN was employed to perform the numerical simulations of nonlinear frac- tional dynamics based on various types of FDE, the obtained results were compared with other numerical methods, such as the spectral collocation method, meshless method, and reproducing kernel method, to demonstrate the feasibility of the proposed approach.

The stability of impulsive complex-valued BAM FANN with time-varying delays was studied in Ref. [23]. In this work, the Laplace transform of the MittagLeffler function was obtained, and the Mittag-Leffler stability of the Caputo fractional derivative was proved.

For homogeneity reasons, each table presented in this paper shows the ANN architectures that involve: time delay, time varying-delays, multiple delays, mixed delays, and leakage delay, followed by the architecture of the ANN.

Table 1 shows the overview of methods used to solve DE and FDE applying ANN involved with FC. The types of the differential equation, the referenced work, the method to solve the DE or FDE, the ANNs architectures, as well as the fractional derivative used, are shown in Table 1, as follows:

According to the bibliographic analysis developed in this manuscript, we can affirm that the most used method to solve FDE and Delayed FDE is the Laplace transform method, followed by the Adams-BashforthMoulton method, both of them mainly used the Caputo derivative.

\subsection{Brief analysis about numerical methods}

The interest in applying and solving fractional differential equations (FDEs) has increased in the last decades. Methods such as Laplace/Sumudu perturbation $[62,67,68,98,114,122,123]$, Adomian decomposition $[46,124,125]$, homotopy perturbation [126] or decomposition have been used to reach this objective. However, these methods are faced with the convergency, the stability, ability to handle strong non-linearities [127] even the presence of a persistent memory [128].

For this reason, linear multistep methods are a powerful option to solve fractional differential equations. The Adams-Bashforth method (ABM) is an efficient numerical scheme that converges toward the exact solution. One can see several types of research whose numerical results were reached by $\mathrm{ABM}[3,24,33,34,42,44$, $45,82,95,97]$. It can lead to non-local, non-singular kernel fractional derivatives [129]. However, ABM requires several floating-point operations [128], and this has limitations because of the Lagrange polynomial. Dealing with these limitations, Atangana and Araz [130] developed a numerical scheme based on Newton polynomial, which seems to be accurate.

Therefore, the proposal of numerical schemes for solving FDEs is still an open field for new accurate proposals with the ability to handle strong non-linearities with the least computational effort. 


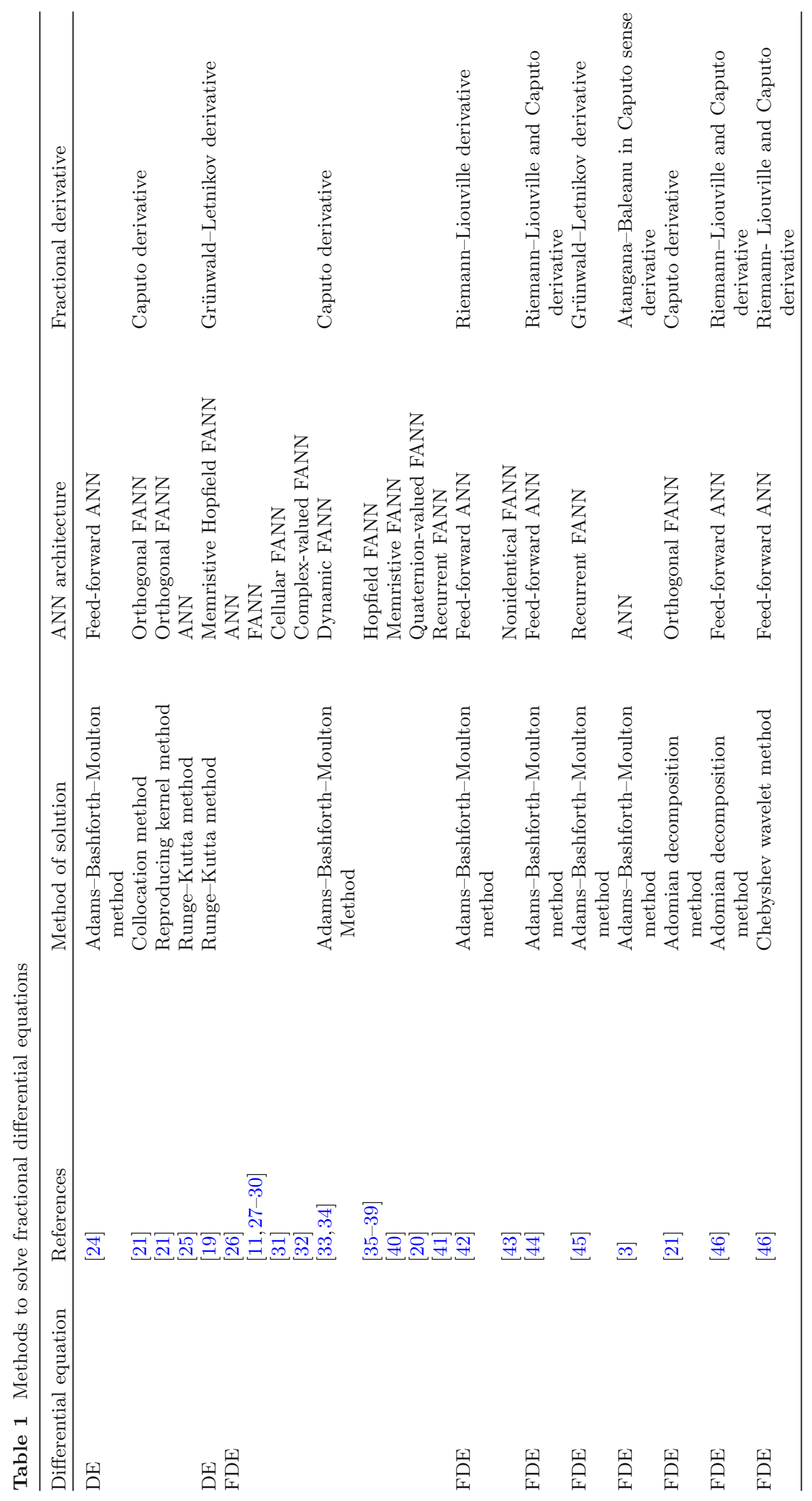




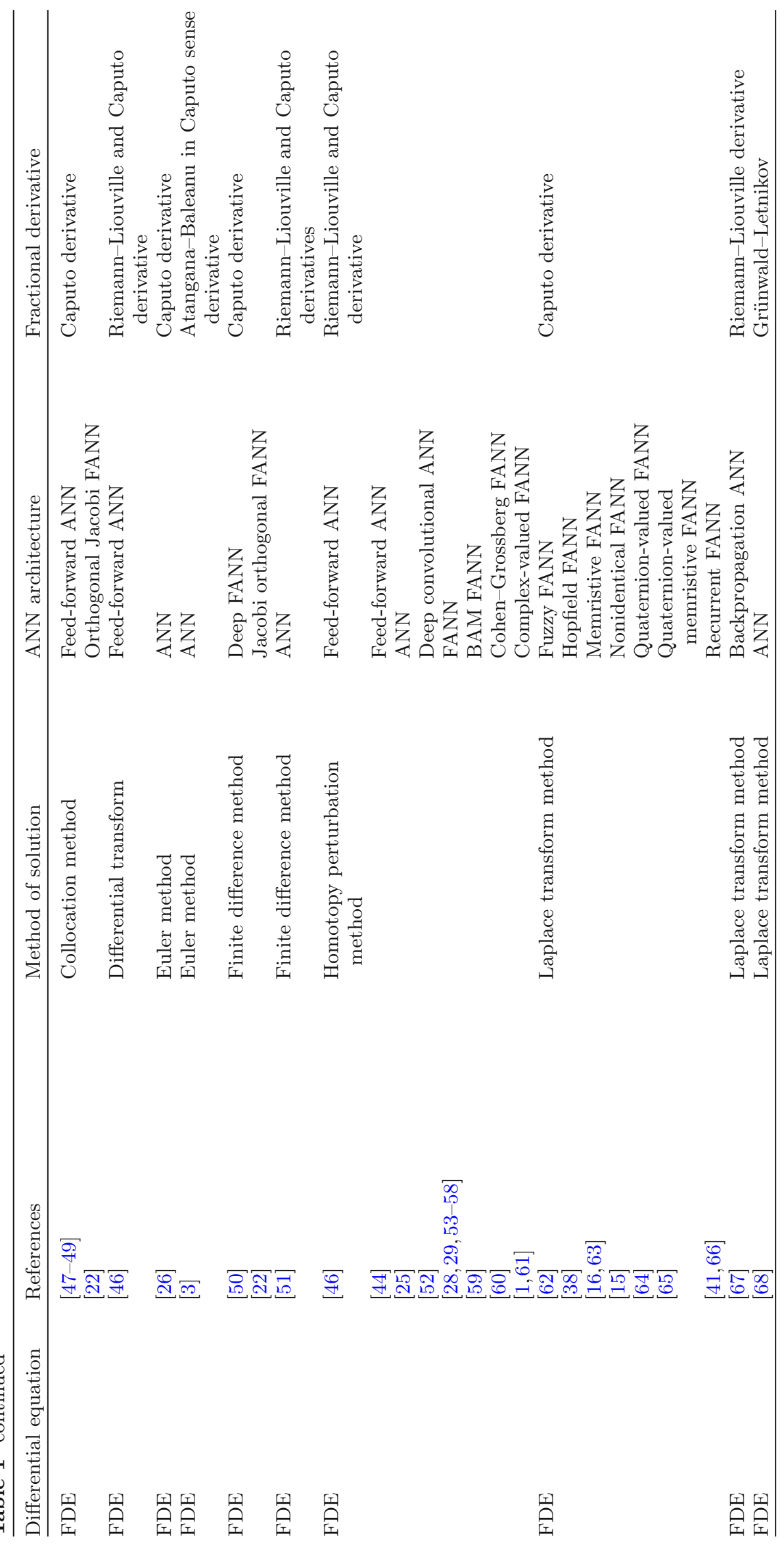




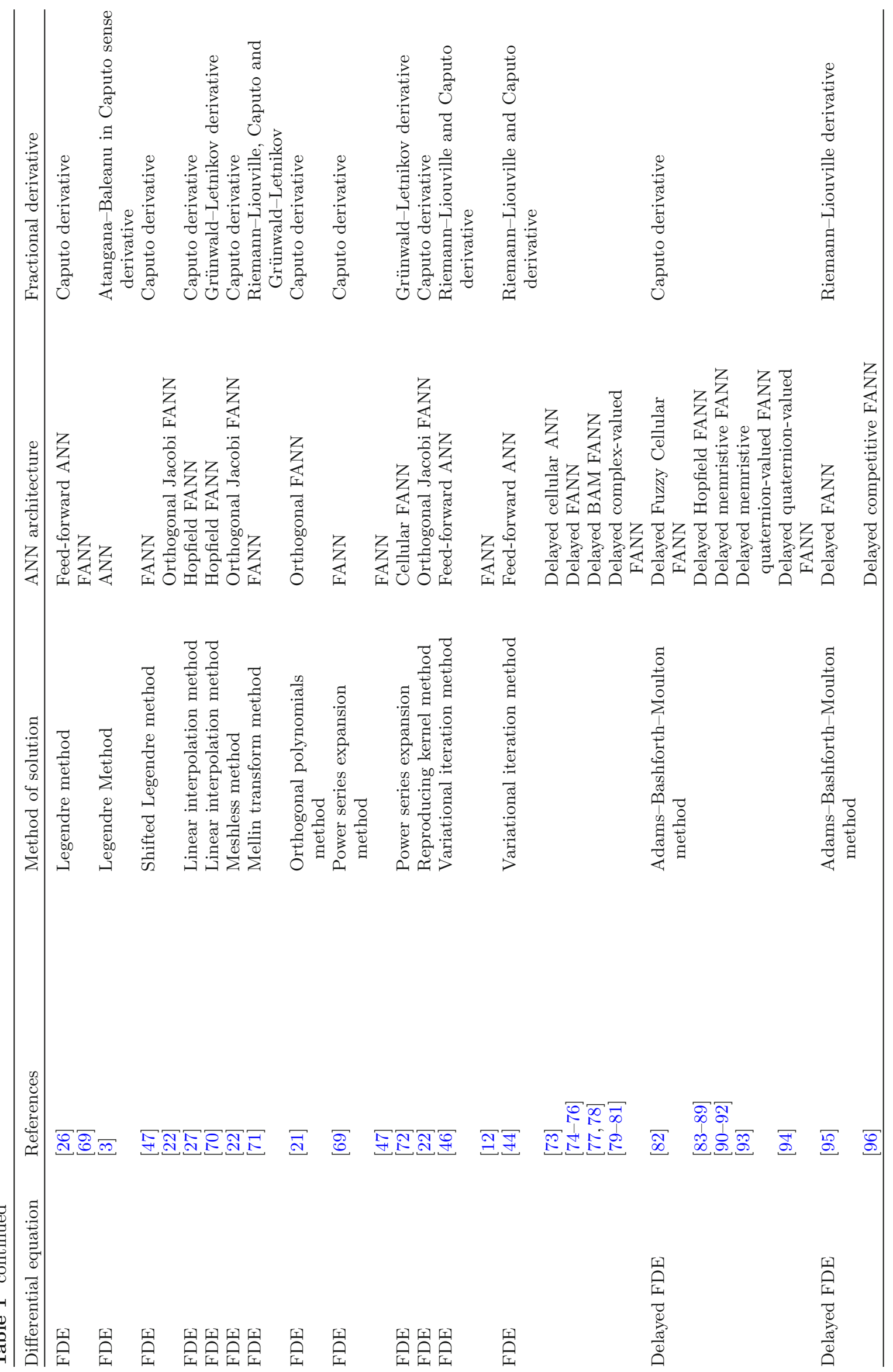




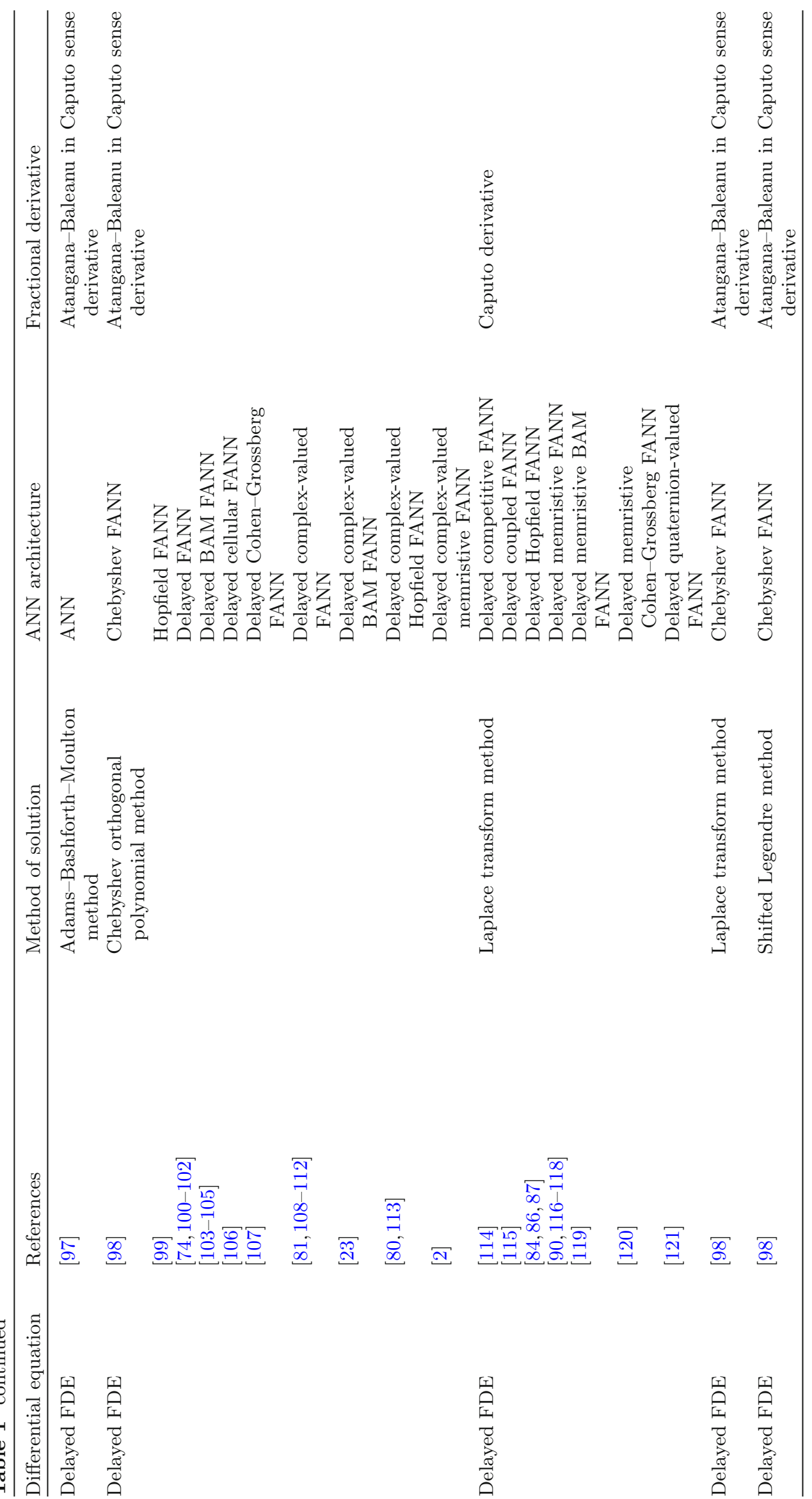




\section{Optimization algorithms for training artificial neural networks}

This section presents the works found in the review of the state of the art where optimization algorithms are employed for the training of ANN involved with FC. First, the works with a fractional approach are described. Afterwards, the proposals under integerorder operators are described.

\section{Optimization algorithms with fractional approach}

In literature, there were found six works where the fractional GD algorithm was implemented, four works based on fractional BP algorithm, and one research where a Darwinian particle swarm optimization algorithm of fractional-order was developed, all of them were used for the training of ANN involved with FC. Next, these works are discussed.

\section{Gradient descent algorithm (GD)}

In Ref. [131], the author implemented a fractional GD algorithm to derive the fractional back-propagation through time (FBPTT) algorithm for recurrent ANN, based on the Riemann-Liouville derivative; this fractional algorithm was able to solve three estimation problems, namely: nonlinear system identification, classification of pattern and Mackey-Glass chaotic time series prediction, outperforming the conventional backpropagation through time performance.

Other interesting works were proposed in Refs. $[132,133]$. They got a fractional GD back-propagation method based on the Caputo derivative for training an ANN [132] and deep BP ANN [133]. They derived the error function monotonicity, the proposed algorithms presented weak convergence, and numerical simulations demonstrated the competitive performance of the presented fractional models. Compared with classical integer-order models, the fractional models showed significant advantages of memory storage and hereditary characteristics. The authors in Ref. [133] carried out a comparison between two different methods to test their performances, the first was an ordinary BP $\mathrm{ANN}$, and the second one was a fractional-order deep BP ANN. The results of the training and testing showed that fractional-order deep BP ANN has a better performance.

Afterward, as the fractional-order gradient could not converge to the real extreme point. In Ref. [134], the authors designed a new fractional-order gradient method based on Caputo derivative for the BP of convolutional ANN. In this work, the parameters within the layers were updated using the fractional gradient method, but propagations between layers used integer gradients to keep the chain rule. In fact, the proposed fractional-order gradient method guaranteed the convergence to a real extreme point, fast convergence, high accuracy, and ability to escape local optimal point in ANN when compared with integer-order ANN.
Also, Chen et al. [135] implemented an adaptive fractional-order BP ANN. This technique uses the population extremal optimization, as well as the fractionalorder GD training algorithms. The method was developed to solve handwritten digit recognition problems. Population extremal optimization algorithms were used to optimize the initial connection weight parameters, and the fractional GD was used to update these connection weight parameters.

Finally, a FANN was proposed in Ref. [136] for the identification of three different systems. The FANN was trained by using the GD algorithm and the GrünwaldLetnikov derivative. In this work, the three systems that we identified are two benchmark systems and one experimental system. The benchmark systems are a hairdryer, consisting of a mesh of resistor wires heating the air at the entrance of a pipeline, and a hysteresis model consisting of the Bouc-Wen model used to represent hysteresis effects in mechanical engineering. Besides, the experimental acoustic duct system is based on an acoustic waves pipeline. The results demonstrated that the fractional gradient descent algorithm allowed accurate estimations with a reduced number of parameters, compared with other works found in the literature where the gradient descent algorithm of integer-order was employed.

\section{Backpropagation algorithm (BP)}

In Ref. [137], the authors developed a Hopfield FANN in the form of an analog circuit. To carried out this, they used factorial, as well as steepest descent fractional approaches.

The authors in Ref. [138] developed a fractional-order BP ANN for improving the performance of the ordinary first-order BP ANN, which was trained by an improved fractional-order steepest descent method. The proposed approach showed to be capable of finding the global optimal solutions. The BPFANN was compared with a classic first-order BPANN by means of an example function approximation, fractional-order multi-scale global optimization, and two comparative performances with real data, involving Grünwald-Letnikov derivative. The BPFANN was compared with a classic firstorder BPANN. To carry out the methods comparison, they were used an approximation function, a fractionalorder multi-scale global optimization method, and two real data sets. The FANN was developed involving the Grünwald-Letnikov derivative. The BPFANN was superior to the classic first-order BPANN in terms of finding the global optimal solution.

Additionally, in Ref. [139], the authors implemented a fractional observer ANN for high complex fractionalorder nonlinear systems, involving the Caputo derivative, for estimating the state variables of a fractionalorder nonlinear chaotic system with the unknown dynamic model. A new fractional error back-propagation learning algorithm was developed to adapt the weights of the ANN. This method could eliminate the effect of uncertainties and unmodeled dynamics of the system, 
showing a fast convergence. The fractional observer was better than other observers of integer-order in terms of accuracies for fractional systems, distinguishing external disturbances, and modeling uncertainties more efficiently.

\section{Darwinian particle swarm optimization algorithm (FO-PSO)}

In Ref. [24], the authors developed a feed-forward ANN optimized by applying a fractional-order Darwinian particle swarm optimization algorithm (FO-DPSO) to calculate better solutions to the nonlinear second-order ordinary differential equations representing the corneal shape model (CSM). The authors used the GrünwaldLetnikov derivative. Adams's numerical solver was used as the reference solution. PSO-DPSO was compared with the hybridization between the PSO algorithm and the Active set algorithm (PSO-ASA). The result showed that ANN-based FO-DPSO was more accurate in the solutions with fewer residual errors. Performance matrices like MAD, TIC, and ENSE were used to test the efficiency of the proposed approach, demonstrating that the proposed methodology was better in terms of less number of function evaluations, mean time value, ENSE, TIC, MAD. FO-DPSO was an excellent technique for tuning the unknown weights involved in the solution designed with ANNs.

\section{Optimization algorithms with classical approach}

From the bibliographic review, there were found seven works where the classical GD has been used as an optimization algorithm for training the ANN involved with FC. In two research works, the classical BP algorithm was proposed. Moreover, in six works, the classical Levenberg-Marquardt algorithm (LM) was implemented for training the ANNs. On the other hand, some other classical optimization algorithms have been employed with this purpose, such as interior point algorithm (IPA), genetic algorithm hybridized with pattern search algorithm (GA-PS), sequential quadratic programming algorithm (SQP), BroydenFletcher-Goldfarb-Shanno algorithm (BFGS), chaotic differential evolution algorithm (CDE), simulated annealing algorithm (SA), particle swarm optimization algorithm (PSO), (SA) hybrid with particle swarm optimization algorithm (PSO) obtaining the (PSOSA) algorithm, particle swarm optimization algorithm hybrid with enhanced fruit fly algorithm (PSO-EFF), particle swarm optimization algorithm hybrid with artificial bee colony algorithm (PSO-ABC), and stochastic inertia weight particle swarm optimization (SIWPSO) algorithm.

All the above-mentioned works are going to be explained in detail as follows:

\section{Gradient descent algorithm (GD)}

Firstly, in Ref. [67], the authors proposed a fractional PID controller with self-tuning parameters based on BP ANN. The discretization method and the design method of the controller were discussed. The authors used the Riemann-Liouville derivative to develop the fractional controller. The controller parameters were tuned by an ANN, which was optimized by the GD optimization algorithm. The fractional PID controller was more flexible than the ordinary. The fractional controller allowed the authors to adjust better its dynamical properties than the ordinary PID. Moreover, the fractional PID controller with a self-tuning parameter based on a BP ANN kept features of a normal fractional PID controller. It has better flexibility because an ANN was introduced to develop the self-tuning controller.

Subsequently, a set of fractional differential equations of initial value problems constructed from cosine basis functions with adjustable parameters were solved in Ref. [140] using an ANN and the Caputo derivative. Numerical solutions were obtained for a single FDE, as well as for systems of coupled FDE. The numerical solutions were obtained by training the ANN repeatedly by means of the GD algorithm. Numerical results were in good agreement with the exact solutions of the FDE.

Similarly, Jafarian in Ref. [141] employed the Caputo derivative over a bounded domain to approximate series solutions of a class of initial value FDE. The ANN was used to solve a fractional type ordinary DE. The original differential equation was transformed into a minimization problem, which was solved using an accurate ANN model for computing the parameters accurately. The authors achieved this using the GD procedure for training a feed-forward ANN. The proposed algorithm was an efficient tool for finding the unknown series coefficients. The obtained simulation results were compared with the exact solutions, Chebyshev wavelet method, and the Homotopy perturbation method, reported in the literature. Finally, the authors demonstrated that numerical simulations carried out by the ANN trained using the GD algorithm were similar to the solutions reported in the literature.

On the other hand, a Chebyshev functional link ANN was proposed by Kheyrinataj and Nazemi in Ref. [98] to model linear and nonlinear delay fractional optimal control problems involving Atangana-Baleanu derivative. The trial solutions were approximated by applying the Chebyshev functional link ANN, which was trained using the GD algorithm. This work presented the first application of delay fractional optimal control with mixed control-state constraints employing a fractionalorder derivative with the nonlocal and nonsingular kernel using the Chebysehv functional link ANN approach.

Besides, Antil in Ref. [50] proposed a deep FANN for the time-discretization of a fractional in time nonlinear ordinary DE, employing Caputo derivative. The fractional ordinary DE was minimized by the learning algorithm, solving several issues, such as network instability, vanishing, exploding gradients, long training times, and inability to approximate non-smooth functions. Keep- 
ing track of history in this manner improved the vanishing gradient problem and could potentially strengthen feature propagation. It was numerically illustrated the improvement in the vanishing gradient issue using of the proposed deep FANN allowing a better learning ability. The deep FANN was better capable of passing information across the network layers maintaining the relative gradient magnitude across the layers, compared to the standard deep ANN and standard Residual ANN. The deep FANN improved the vanishing gradient issue due to the memory effect, and it handled much better the nonsmooth data due to the network's ability to approximate non-smooth functions.

Finally, the authors in Ref. [49] tested the ability of a perceptron ANN to approximate functions to solve fractional infinite-horizon optimal control problems involving Riemann-Liouville and Caputo derivatives. The authors used the GD algorithm for training the ANN. There were no other reported works about solving this problem in the literature. Numerical simulations demonstrated the feasibility and efficiency of using the proposed method for solving optimal control problems.

\section{Backpropagation algorithm (BP)}

The BP algorithm was used for training a Master-Slave FANN based on Caputo derivative in Ref. [27]. The master network was composed of two Hopfield networks, meanwhile and the slave network was a BP network, doing the BP the system error. The Master-Slave FANN showed to have the highest asymptotic convergence rate and the smallest system error compared with Master-Slave ANN of integer-order.

Moreover, in Ref. [142] the authors found the numerical solution of FDE by employing the Chebyshev ANN, Riemann-Liouville, and Caputo derivatives. BP algorithm was used to train the feed-forward Chebyshev ANN. The accuracy of the proposed method was shown by comparing the analytical solutions with the numerical results. The obtained results showed a good agreement with analytical solutions. The comparison results showed that the Chebyshev ANN is a capable tool for solving linear and nonlinear problems.

Nouh [143] modeled the fractional polytropic gas spheres, which have several applications in physics, astrophysics, engineering, and so on. Thus, the fractional Lane-Emden differential equations of the fractional polytropic gas spheres phenomena were solved employing ANN-based on back-propagation training algorithm, reaching the training of the ANN with small errors predicting the values of fractional Lane-Emden functions.

\section{Levenberg-Marquardt algorithm (LM)}

Firstly, Efe and Member in Ref. [144] developed an ana$\log$ PID controller using an approach of feed-forward ANN. The implementation of fractional-order operators in the PID controller was discussed for establishing a robust control for applications in unmanned aerial vehicles (UAVs). The ANN was trained to provide the coefficients of a finite impulse response (FIR) of approximation type. Some trajectories were described properly by the FIR controller, and the feed-forward ANN, which was trained by the LM algorithm.

Next, in Ref. [145] the authors developed a new method to detect unilateral hearing loss (left-sided and right-sided); the fractional Fourier transform (FRFT) was employed to detect hearing loss more efficiently and accurately. The classifier was a feed-forward ANN trained by the LM algorithm. Some magnetic resonance images were obtained from studies with real patients. The combination of fractional Fourier transform, the principal component analysis, and the neural network as the classifier, showed accuracies higher than $95 \%$ concerning the experimental data [145].

On the other hand, Zúniga-Aguilar [3,97] used an ANN to get the approximated solution of fractional differential equations of Atangana-Baleanu type in Caputo sense with delay and without delay, respectively. In both cases, the network's parameters optimization was carried out using the LM algorithm. The results of both ANNs were compared with the analytical solutions and the numerical simulations obtained through the Adams-Bashforth-Moulton method. Different performance indices were calculated to show the effectiveness of the ANNs. The ANN's were able to achieve approximate solutions with good precision and fast convergence.

Subsequently, Kadam et al. developed the artificial ANN approximation of fractional derivative operators such as Grünwald-Letnikov and Caputo fractional derivatives. LM algorithm was used for training the ANN, considering the mean squared error between the outputs of derivatives and the approximations for validation. Thus, the approximations were computationally fast when compared with the numerical evaluation of fractional-order derivatives.

In a recent paper [21], the authors showed the design of a single layer orthogonal ANN for approximating the solutions of different types of Lane-Emden equations in the Caputo sense. The fractional-order Legendre functions in Caputo sense were used as the hidden layer activation function, while the LM algorithm was used to train the ANN. The obtained results were compared with some other numerical methods and with the exact solution, showing that the proposed orthogonal ANN was accurate and feasible.

Finally, Hadian Rasanan in Ref. [22] implemented a fractional ANN. The authors used fractional-order Jacobi functions as the activation function of the hidden layer. And the identity function was used as the activation function of the output layer. The goal of this work was to approximate the solution of FDE and partial FDE involving Caputo derivative. LM was the training algorithm employed. Thereby, the proposed ANN had the ability to reach high accuracy with few neurons. The effectiveness of the proposed ANN was validated applying linear and nonlinear fractional dynamics. The numerical results were compared with the 
results obtained from other ANN and some numerical experiments, demonstrating that the proposed model is accurate, fast, and feasible.

\section{Interior point algorithm (IPA)}

Regarding the use of the IPA algorithm for the training of ANN involved with FC, Asif in Ref. [12] found the solution of fractional systems governed by the initial value problems of the Bagley-Torvik equation employing a FANN trained with the IPA algorithm via Caputo and Riemann-Liouville derivatives. The designed method was evaluated on different initial value problems of the equation. A comparison between the proposed method and several available criteria, such as an exact solution; Podlubny numerical techniques; an analytical solver based on variational iteration method; and a reported solution of stochastic solvers based on hybrid approaches, allowed to verify the effectiveness of the designed method. Concluding, this efficient computational technique based on FANN, optimized with IPA, was able to find the solutions of different variants of Bagley-Torvik equations in a more accurate way than other stochastic techniques.

Also, in Ref. [146] authors found the approximate solutions of nonlinear quadratic systems based on Riccati equations of fractional-order by means of FANN trained with IPA algorithm. The obtained results were compared with the exact solutions proving the effectiveness of the proposal. This method matched more closely with the standard solution obtained from AdamsBashforth-Moulton method than the modified homotopy perturbation method and enhanced homotopy perturbation method. The average time consumed by the IPA for a run was lower that than other stochastic techniques based on the PSO and GA algorithms.

\section{Genetic algorithm and pattern search (GA-PS)}

A new method to train ANN involved with FC was developed in [147], where a fractional-order system represented by Bagley-Torvik equation was solved by means of feed-forward ANN. This new method is based on evolutionary computational, and it is called the GA algorithm hybrid with the PS technique. Besides, in this work, the Riemann-Liouville derivative was used. The proposed method was successfully applied to different forms of the equation, and the results were compared with a standard approximate analytic solution, stochastic numerical solvers, and exact solutions. The GA, PS, and Ga hybrid with PS (GA-PS) optimizer algorithms were compared against each other for evaluating the performances of the training algorithms, obtaining the best results with the GA-PS algorithm.

\section{Sequential quadratic programming algorithm (SQP)}

In Ref. [44], the authors applied a feed-forward ANN and SQP algorithm for the training of weights to obtain the solution of nonlinear quadratic Riccati FDE involv- ing the Riemann-Liouville and the Caputo derivatives. The obtained results with the proposed methodology coincided with the exact solution based on the AdamsBashforth-Moulton technique. Even, the results were more accurate than the obtained with both the modified homotopy perturbation and the enhanced Homotopy perturbation methods showing the effectiveness of the proposed scheme.

\section{Broyden-Fletcher-Goldfarb-Shanno algorithm (BFGS)}

An adaptive fractional-order PID controller using ANN was designed based on auto-tuning neurons, involving Caputo derivative in [51]. The Nelder-Mead simplex search method and BFGS algorithm were used for the parameters tuning. The proposed controller was more robust in comparison with conventional controllers [51].

Also, the BFGS quasi-Newton algorithm was employed for training a perceptron ANN involving Caputo derivative in Ref. [26] and [148] to solve FDE and approximate the solution of a fractional optimal control problem, respectively. In [26], the authors validated their method by solving different types of multiterm FEEs. In Ref. [148], the authors validated their proposal by comparing their results with other investigations found in the literature.

\section{Chaotic differential evolution algorithm (CDE)}

In Ref. [149], the authors implemented a wind turbine pitch control for regulating the speed of the rotor and power production. The authors proposed a fractionalorder PID combined with a radial basis function ANN to improve the performance and alleviating the mechanical loads. The ANN was trained with the CDE algorithm. The fractional-order PID presented better performance and robustness when comparing with other controllers. Moreover, the FOPID controller alleviated mechanical loads in a better way, compared with other control techniques, such as the PID, PI, radial basis function PI, and fractional-order PI controller.

\section{Simulated annealing algorithm (SA)}

The chaotic behavior of fractional-order Chua's system was studied by means of fractional Laplace transform, the activation function for the ANN was the Mexican hat wavelet. The ANN was trained using SA and the analytical solution for the system could be approximated. The accurate approximated solutions, the phase plots of the Lyapunov exponent spectrum, and bifurcation maps of the dynamical evolution of fractional Chua's system were achieved. Mexican Hat Waveletbased ANN with SA and fifth-fourth Runge-Kutta method, were proposed to attain the solutions of fractional Chua's model. Using Caputo derivative, cubic nonlinear was solved efficiently and the Chua's circuit variables were optimized for different fractional values, in [25]. 
Subsequently, in Ref. [150] the authors found the numerical solution of delayed FDE based on the application of neural minimization using Chebyshev simulated annealing ANN and Legendre simulated annealing ANN. Chebyshev and Legendre polynomials were used with SA to reduce mean square error and get more accurate numerical approximations. This work was based on the functional link ANN with optimization through thermal minimization. Caputo's definition was employed for calculating the fractional derivative in the subsequent procedure, and the learning methodology used in this work was the SA algorithm. The obtained results were validated using various experiments, numerically. And graphically with error analysis to demonstrate the accuracy and efficiency of the proposed approach.

\section{Particle swarm optimization (PSO)}

In Ref. [34], the authors presented a fractional-order dynamic ANN trained by the PSO algorithm for identifying the Damavand tokamak plasma behavior using the Caputo derivative. The system stability was demonstrated based on the Lyapunov-like analysis. The performance of the proposed approach was compared with experimental data and the integer-order ANN approach. The comparison results showed that the fractional-order dynamic ANN was higher accurate than the dynamic ANN of integer-order.

Zhang and Yang in Ref. [151], studied the optimal quasi-synchronization problem for delayed memristive FANN involving Caputo derivative. Fractional-order inequalities and aperiodically intermittent controllers were proposed to guarantee the quasi-synchronization of the system. Mittag-Leffler function allowed to get the stability result of the fractional-order delayed system. Synchronization of the delayed memrisitve delayed FANN was ensured thanks to matrix inequalities. The control parameters were optimized, and the smaller control energy was obtained applying the PSO algorithm. Simulation examples showed the correctness of the proposed method.

Aslipour and Yazdizadehin in Ref. [34] optimized a dynamic FANN involving Caputo derivative. The authors used the PSO algorithm to identify the behavior of a wind turbine in operation. The results obtained from dynamic FANN were compared with the results obtained from dynamic ANN of integer-order, highlighting that the fractional method was more accurate.

\section{Particle swarm optimization-simulated annealing algorithm (PSO-SA)}

In Ref. [46], the authors developed a feed-forward ANN used for approximating the solution of nonlinear Riccati FDE using Riemann-Liouville and Caputo derivatives. The network training was carried out using the particle swarm optimization algorithm (PSO) hybridized with the simulated annealing algorithm (SA). The results were compared with the standard approximate analyti- cal method, the stochastic numerical solvers, and exact solutions. The weights training was proved with other stochastic algorithms, such as SA, GA, GA hybridized with SA (GA-SA), PSO, and PSO hybridized with SA (PSO-SA). The best optimization results were obtained by PSO-SA algorithm.

In Ref. [42], the PSO-SA algorithm was employed for the training of a feed-forward ANN. In this case, the ANN approximated the mathematical model of FDE using Riemann-Liouville and Grünwald-Letnikov derivatives. Comparison between the obtained results and the available exact solutions, analytic solutions, and standard numerical techniques (including both deterministic and stochastic approaches) was carried out, showing that This approach was properly employed to solve different problems associated with linear and nonlinear ordinary FDE. The training of weights was implemented using PSO, GA, GA-SA, and SA algorithms, but the best results were obtained using the PSO-SA algorithm.

\section{Particle swarm optimization and enhanced fruit fly (PSO-EFF)}

In Ref. [68], authors developed a nonlinear neural fractional-order proportional integral derivative controller based on ANN and involving the GrunwaldLetnikov derivative, applied to the motion control of a nonholonomic differential drive of a mobile robot. The hybridization of a modified adaptive PSO and the EFF optimization algorithms were used for tuning the parameters of the fractional-order PID controller based on ANN. The fractional-order PID controller decreased the control signals that drive differential drive mobile robot motors by approximately $45 \%$ compared with the PID based on ANN of integer-order, and thus reduced the energy consumption in circular trajectories. Numerical simulations demonstrated that the performance of the designed fractional controller was excellent compared with nonlinear controllers of integer-order on the trajectory tracking of the differential drive mobile robot with different trajectories as study cases.

\section{Particle swarm optimization and artificial bee colony (PSO-ABC)}

In the current year, Mohammadzadeh and Kayacan in [152] developed an adaptive fractional-order fuzzy control method in the sense of Caputo's definition to control the frequency in an $\mathrm{AC}$ microgrid (MG). A sequential general type-2 fuzzy system based on the radial basis ANN was presented for online modeling of the frequency response of the MG. The parameters were optimized using of a hybridized approach between PSO and $\mathrm{ABC}$ algorithms. The learning algorithm was examined using white noise as the control input. It was demonstrated that the proposed identification scheme results were in good performance even in a noisy environment. The results had robust performance in the presence of variation of solar radiation, wind speed, load distur- 
bance, and time-varying dynamics of the other units of MG. The proposed control approach was compared with the conventional PI controller, classic fuzzy, and PSO-fuzzy PI controllers. The results showed that the proposed scheme has better performance.

\section{Stochastic inertia weight particle swarm optimization (SIWPSO)}

Optimization of synchronization for delayed memristive FANN was investigated in Ref. [153], involving Caputo derivative. It was designed an appropriate controller where the drive system was able to synchronize with the response system. Synchronization conditions were obtained thanks to the linear matrix inequality, along with fractional-order Lyapunov methods. The FANN was trained using of the SIWPSO algorithm, the target function was the minimal sum of control energy expressed by the integral square error-index, where the Riemann-Liouville derivative was used to get the approximated value of the target function. It was obtained a better controller with low control energy and integral square error (ISE) index. The optimal control parameters of the proposed model were obtained by computing the SIWPSO algorithm, which was an improved intelligent algorithm. A simulation was provided to demonstrate the feasibility of the proposed results.

\section{Synchronization and stabilization of ANN involved with FC}

The FC applied to ANNs allows simulating systems more accurately than by the classical approach, due to the FC properties as the nonlocality and the memory description of FC. From the bibliographic review, we can affirm that the main applications of FC applied to ANN are system stabilization, systems synchronization, and the training of these systems through optimization algorithms.

This section is organized as follows: first, the control strategies carried out to guarantee the synchronization and stabilization of ANN involved with FC are summarized. Second, other examples of stabilization reached using $\mathrm{FC}$ on ANN are overviewed.

\subsection{Control strategies employed to synchronize and stabilize ANN involved with FC}

Several authors have used control strategies to synchronize and stabilize different architectures of ANN involved with FC. A brief overview of them will be described as follows:

On the one hand, in $[11,13,55,154-161]$ authors employed adaptive control to reach: projective, mixed projective synchronization, and synchronization using the Caputo derivative.
On the other hand, sliding mode control was employed in $[15,58,162-166]$ to reach: projective, global projective, mixed projective synchronization, and synchronization using the Caputo and Riemann-Liouville derivatives.

Also, feedback control was found in several works on ANN involving FC. This control strategy was applied in Ref. [20,43,53,56,59,61,90-92,96,116-119, 154,167-183], and aiding to achieve: hybrid projective, finite-time projective, projective, quasi-projective, quasi-uniform synchronization, stability, finite-time stability, synchronization, and global stability using Caputo and Riemann-Liouville derivatives.

Other control strategies, such as adaptive control, impulsive control, or washout filter control were employed in $[101,184-187]$ to attain: projective, adaptive, global, chaotic synchronization, and global stability, using the Caputo derivative and GrünwaldLetnikov derivatives.

The most used fractional derivative to guarantee the synchronization of these types of systems is the Caputo derivative, followed by the Riemann-Liouville and the Grünwald-Letnikov derivatives, respectively. We found that the Grünwald-Letnikov derivative was only used in one work [187].

The information given in the above sections is summarized in Tables 2 and 3. The tables show the relationship among the control strategy, ANN architecture, the fractional derivative, and the goal achieved (application), as follows:

\subsection{ANN involved with FC to reach stability}

Several authors in literature stabilized ANN involved with FC, thanks to the FDE employed in these systems. In this sense, different types of fractional derivatives have been used to reach systems stabilization. Following, we will present some related works to systems stabilization using ANN involved with FC.

Caputo derivative was employed to reach the exponential stability, in Refs. [32,171,232], the uniform stability in $[76,79,233-242]$, the finite-time stability in Refs. $[75,85,103,175,243-248]$, the stability and bifurcation in Refs. [36, 37, 41,73,74,77, 80, 108, 249-251], and the quasi-uniform stability, fractional input stability, global stability, and global periodicity in Refs. [252254], respectively.

Similarly, the Caputo and Riemann-Liouville derivatives were employed to reach: the global stability in Refs. [16, 28, 38, 59, 62, 86, 95, 117, 174, 180, 185, 255-259], the stability in Refs. $[35,41,54,57,66,84,87,113,155$, 260-268].

Also, the Riemann-Liouville derivative was used to reach synchronization stability in Refs. $[252-254,269$, 270].

Concluding, the Caputo derivative is the most used fractional derivative for investigating the stability of the ANN involved with FC, followed by the RiemannLiouville derivative. Nowadays, Caputo and Riemann- 
Table 2 Control strategies employed to synchronize and stabilize ANN involved with FC

\begin{tabular}{|c|c|c|c|c|}
\hline $\begin{array}{l}\text { Control } \\
\text { strategy } \\
\end{array}$ & References & ANN architecture & Fractional derivative & Application \\
\hline \multirow{16}{*}{$\begin{array}{l}\text { Adaptive } \\
\text { control }\end{array}$} & {$[188]$} & $\mathrm{ANN}$ & & Synchronization \\
\hline & {$[156]$} & Chebyshev ANN & & Synchronization \\
\hline & {$[161]$} & Fuzzy ANN & & Synchronization \\
\hline & {$[13,160]$} & FANN & & $\begin{array}{c}\text { Global projective } \\
\text { synchronization }\end{array}$ \\
\hline & {$[11,154]$} & FANN & & Global synchronization \\
\hline & {$[55,155]$} & FANN & & Synchronization \\
\hline & {$[189]$} & Chaotic FANN & & $\begin{array}{l}\text { Approximation and } \\
\text { stability }\end{array}$ \\
\hline & {$[82]$} & Fuzzy cellular FANN & Caputo derivative & $\begin{array}{l}\text { Global stability and } \\
\text { synchronization }\end{array}$ \\
\hline & {$[159]$} & Memristive FANN & & $\begin{array}{l}\text { Projective } \\
\text { synchronization }\end{array}$ \\
\hline & {$[190]$} & $\begin{array}{l}\text { Memristive FANN with } \\
\text { leakage delay }\end{array}$ & & Synchronization \\
\hline & {$[191]$} & $\begin{array}{l}\text { Radial basis function } \\
\text { FANN }\end{array}$ & & Synchronization \\
\hline & {$[158]$} & Delayed FANN & & $\begin{array}{r}\text { Mixed projective } \\
\text { synchronization }\end{array}$ \\
\hline & {$[192]$} & Delayed BAM FANN & & $\begin{array}{l}\text { Stability and } \\
\text { synchronization }\end{array}$ \\
\hline & {$[157]$} & Delayed fuzzy FANN & & $\begin{array}{l}\text { Projective } \\
\text { synchronization }\end{array}$ \\
\hline & {$[109]$} & $\begin{array}{l}\text { Delayed } \\
\text { complex-valued } \\
\text { FANN }\end{array}$ & & $\begin{array}{l}\text { Quasi-projective and } \\
\text { complete } \\
\text { synchronization }\end{array}$ \\
\hline & {$[193]$} & $\begin{array}{l}\text { Delayed competitive } \\
\text { FANN }\end{array}$ & & $\begin{array}{l}\text { Projective } \\
\text { Synchronization }\end{array}$ \\
\hline \multirow{8}{*}{$\begin{array}{l}\text { Adaptive } \\
\text { sliding mode } \\
\text { control }\end{array}$} & {$[194]$} & Hopfield FANN & Caputo derivative & Stability \\
\hline & {$[195]$} & Delayed fuzzy FANN & & $\begin{array}{l}\text { Projective } \\
\text { synchronization }\end{array}$ \\
\hline & {$[52]$} & $\begin{array}{l}\text { Deep convolutional } \\
\text { ANN }\end{array}$ & & Robotic manipulators \\
\hline & {$[196]$} & Deep recurrent ANN & & Synchronization \\
\hline & {$[165]$} & $\begin{array}{l}\text { Radial basis function } \\
\text { ANN }\end{array}$ & & Stability \\
\hline & {$[197]$} & $\begin{array}{l}\text { Radial basis function } \\
\text { ANN }\end{array}$ & & HIV infection model \\
\hline & {$[198,199]$} & Recurrent ANN & & Approximation \\
\hline & [198] & Recurrent ANN & & Stability \\
\hline \multirow{7}{*}{$\begin{array}{l}\text { Sliding Mode } \\
\text { Control }\end{array}$} & {$[58]$} & FANN & Caputo derivative & Synchronization \\
\hline & {$[200]$} & Chaotic FANN & & Syncrhonization \\
\hline & {$[201]$} & $\begin{array}{l}\text { Memristive MAM } \\
\text { FANN }\end{array}$ & & $\begin{array}{l}\text { Fixed-time } \\
\text { synchronization }\end{array}$ \\
\hline & {$[15]$} & Nonidentical FANN & & $\begin{array}{l}\text { Projective } \\
\text { synchronization }\end{array}$ \\
\hline & {$[166]$} & $\begin{array}{l}\text { Delayed Hopfield } \\
\text { FANN }\end{array}$ & & $\begin{array}{l}\text { Adaptive } \\
\text { synchronization }\end{array}$ \\
\hline & {$[162]$} & $\begin{array}{l}\text { Delayed nonidentical } \\
\text { FANN }\end{array}$ & & $\begin{array}{l}\text { Projective } \\
\text { synchronization }\end{array}$ \\
\hline & {$[164]$} & Hopfield FANN & & Finite-time stability \\
\hline
\end{tabular}


Table 2 continued

\begin{tabular}{|c|c|c|c|c|}
\hline $\begin{array}{l}\text { Control } \\
\text { strategy } \\
\end{array}$ & References & ANN architecture & Fractional derivative & Application \\
\hline $\begin{array}{l}\text { Sliding Mode } \\
\text { Control }\end{array}$ & {$[163]$} & Nonidentical FANN & $\begin{array}{l}\text { Riemann-Liouville } \\
\text { Derivative }\end{array}$ & $\begin{array}{r}\text { Global projective } \\
\text { synchronization }\end{array}$ \\
\hline $\begin{array}{l}\text { Sliding Mode } \\
\text { Control }\end{array}$ & {$[19]$} & $\begin{array}{l}\text { Memristive Hopfield } \\
\text { FANN }\end{array}$ & $\begin{array}{l}\text { Grünwald-Letnikov } \\
\text { derivative }\end{array}$ & Synchronization \\
\hline \multirow[t]{2}{*}{$\begin{array}{l}\text { Pinning } \\
\text { Control }\end{array}$} & {$[202]$} & Delayed BAM FANN & Caputo Derivative & $\begin{array}{l}\text { Quasi-pinning } \\
\text { synchronization and } \\
\text { stability }\end{array}$ \\
\hline & {$[107]$} & $\begin{array}{l}\text { Delayed } \\
\text { Cohen-Grossberg } \\
\text { FANN }\end{array}$ & & $\begin{array}{l}\text { Stability and pinning } \\
\text { synchronization }\end{array}$ \\
\hline \multirow{24}{*}{$\begin{array}{l}\text { Feedback } \\
\text { pinning } \\
\text { control }\end{array}$} & {$[40]$} & Memristive FANN & Caputo derivative & Quasi-synchronization \\
\hline & {$[56,171]$} & FANN & & $\begin{array}{l}\text { Stability and } \\
\text { synchronization }\end{array}$ \\
\hline & {$[203]$} & FANN & & $\begin{array}{l}\text { Robust finite-time cost } \\
\text { control }\end{array}$ \\
\hline & {$[29]$} & FANN & & $\begin{array}{l}\text { Hybrid projective } \\
\text { synchronization }\end{array}$ \\
\hline & {$[181]$} & FANN & & Global synchronization \\
\hline & {$[204]$} & Delayed FANN & & Global synchronization \\
\hline & {$[173]$} & Delayed FANN & & Synchronization \\
\hline & {$[90]$} & Delayed FANN & & $\begin{array}{l}\text { Hybrid projective } \\
\text { synchronization }\end{array}$ \\
\hline & {$[154]$} & Delayed FANN & & Synchronization \\
\hline & {$[205]$} & Delayed FANN & & $\begin{array}{l}\text { Projective } \\
\text { synchronization }\end{array}$ \\
\hline & {$[59]$} & BAM FANN & & Stability \\
\hline & {$[206]$} & BAM FANN & & Global Stability \\
\hline & {$[168]$} & Delayed BAM FANN & & Synchronization \\
\hline & {$[78]$} & Delayed BAM FANN & & Global synchronization \\
\hline & {$[207]$} & Delayed BAM FANN & & Finite-time stability \\
\hline & {$[78]$} & Delayed BAM FANN & & Global synchronization \\
\hline & {$[53]$} & Chaotic FANN & & Synchronization \\
\hline & {$[172]$} & Memristive FANN & & Synchronization \\
\hline & {$[118,208]$} & $\begin{array}{l}\text { Delayed memristive } \\
\text { FANN }\end{array}$ & & Synchronization \\
\hline & {$[177]$} & $\begin{array}{l}\text { Delayed memristive } \\
\text { FANN }\end{array}$ & & $\begin{array}{l}\text { Finite-time projective } \\
\text { synchronization }\end{array}$ \\
\hline & {$[209]$} & $\begin{array}{l}\text { Delayed memristive } \\
\text { FANN }\end{array}$ & & $\begin{array}{l}\text { Projective } \\
\text { synchronization }\end{array}$ \\
\hline & {$[117,174]$} & $\begin{array}{l}\text { Delayed memristive } \\
\text { FANN }\end{array}$ & & $\begin{array}{l}\text { Stability and } \\
\text { synchronization }\end{array}$ \\
\hline & {$[210]$} & $\begin{array}{l}\text { Delayed memristive } \\
\text { FANN }\end{array}$ & & Global stability \\
\hline & {$[211]$} & $\begin{array}{l}\text { Delayed memristive } \\
\text { FANN }\end{array}$ & & Synchronization \\
\hline \multirow[t]{3}{*}{$\begin{array}{r}\text { Feedback } \\
\text { Control }\end{array}$} & {$[116]$} & $\begin{array}{l}\text { Delayed memristive } \\
\text { FANN }\end{array}$ & Caputo derivative & $\begin{array}{l}\text { Finite-time } \\
\text { synchronization }\end{array}$ \\
\hline & {$[92]$} & $\begin{array}{l}\text { Delayed memristive } \\
\text { FANN }\end{array}$ & & $\begin{array}{l}\text { Quasi-uniform } \\
\text { synchronization }\end{array}$ \\
\hline & {$[91]$} & $\begin{array}{l}\text { Delayed memristive } \\
\text { FANN }\end{array}$ & & Quasi-synchronization \\
\hline
\end{tabular}


Table 2 continued

\begin{tabular}{|c|c|c|c|c|}
\hline \multirow[t]{25}{*}{$\begin{array}{l}\text { Control } \\
\text { strategy } \\
\end{array}$} & References & ANN architecture & Fractional derivative & Application \\
\hline & {$[180]$} & $\begin{array}{l}\text { Delayed memristive } \\
\text { FANN }\end{array}$ & & Global synchronization \\
\hline & {$[212]$} & $\begin{array}{l}\text { Delayed memristive } \\
\text { FANN }\end{array}$ & & Asymptotic stability \\
\hline & {$[119]$} & $\begin{array}{l}\text { Delayed memristive } \\
\text { BAM FANN }\end{array}$ & & $\begin{array}{l}\text { Finite-time } \\
\text { synchronization }\end{array}$ \\
\hline & {$[182]$} & $\begin{array}{l}\text { Memristive recurrent } \\
\text { FANN }\end{array}$ & & $\begin{array}{l}\text { Finite-time } \\
\text { synchronization }\end{array}$ \\
\hline & {$[93]$} & $\begin{array}{l}\text { Delayed memrisitve } \\
\text { quaternion-valued } \\
\text { FANN }\end{array}$ & & $\begin{array}{l}\text { Synchronization and } \\
\text { stability }\end{array}$ \\
\hline & {$[175]$} & $\begin{array}{l}\text { Cohen-Grossberg } \\
\text { memristive FANN }\end{array}$ & & $\begin{array}{l}\text { Finite-time stability } \\
\text { and synchronization }\end{array}$ \\
\hline & {$[213]$} & $\begin{array}{l}\text { Quaternion-valued } \\
\text { FANN }\end{array}$ & & $\begin{array}{l}\text { Finite-time } \\
\text { synchronization }\end{array}$ \\
\hline & {$[214]$} & $\begin{array}{l}\text { Quaternion-valued } \\
\text { BAM FANN }\end{array}$ & & Synchronization \\
\hline & {$[215]$} & $\begin{array}{l}\text { Quaternion-valued } \\
\text { Memristive FANN }\end{array}$ & & Global stability \\
\hline & {$[20]$} & $\begin{array}{l}\text { Quaternion-valued } \\
\text { FANN }\end{array}$ & & $\begin{array}{l}\text { Global synchronization } \\
\text { and global stability }\end{array}$ \\
\hline & {$[216]$} & $\begin{array}{l}\text { Delayed } \\
\text { quaternion-valued } \\
\text { FANN }\end{array}$ & & Global synchronization \\
\hline & {$[176]$} & $\begin{array}{l}\text { Fuzzy cellular } \\
\text { memristive FANN }\end{array}$ & & $\begin{array}{l}\text { Finite-time stability } \\
\text { and synchronization }\end{array}$ \\
\hline & {$[217]$} & Fuzzy FANN & & Asymptotic stability \\
\hline & {$[218]$} & $\begin{array}{l}\text { Coupled discontinuous } \\
\text { FANN }\end{array}$ & & $\begin{array}{l}\text { Finite-time } \\
\text { synchronization }\end{array}$ \\
\hline & {$[219]$} & $\begin{array}{l}\text { Delayed } \\
\text { non-autonomous } \\
\text { FANN }\end{array}$ & & Synchronization \\
\hline & {$[114]$} & $\begin{array}{l}\text { Delayed competitive } \\
\text { FANN }\end{array}$ & & $\begin{array}{l}\text { Global asymptotic } \\
\text { stability }\end{array}$ \\
\hline & {$[115]$} & $\begin{array}{l}\text { Delayed coupled FANN } \\
\text { with }\end{array}$ & & $\begin{array}{c}\text { Robust asymptotic } \\
\text { synchronization }\end{array}$ \\
\hline & {$[179]$} & $\begin{array}{l}\text { Radial basis function } \\
\text { ANN }\end{array}$ & & $\begin{array}{l}\text { Projective } \\
\text { synchronization }\end{array}$ \\
\hline & {$[61]$} & $\begin{array}{l}\text { Complex-valued } \\
\text { recurrent FANN }\end{array}$ & & $\begin{array}{l}\text { Quasi-projective } \\
\text { synchronization }\end{array}$ \\
\hline & {$[109]$} & $\begin{array}{l}\text { Delayed } \\
\text { complex-valued } \\
\text { FANN }\end{array}$ & & $\begin{array}{l}\text { Quasi-projective and } \\
\text { complete } \\
\text { synchronization }\end{array}$ \\
\hline & {$[167,183]$} & $\begin{array}{l}\text { Delayed } \\
\text { complex-valued } \\
\text { FANN }\end{array}$ & & Synchronization \\
\hline & {$[170]$} & FANN & & Synchronization \\
\hline & {$[220]$} & Delayed FANN & & $\begin{array}{l}\text { Stability and } \\
\text { synchronization }\end{array}$ \\
\hline & {$[221]$} & Delayed FANN & & Synchronization \\
\hline
\end{tabular}


Table 2 continued

\begin{tabular}{|c|c|c|c|c|}
\hline $\begin{array}{l}\text { Control } \\
\text { strategy } \\
\end{array}$ & References & ANN architecture & Fractional derivative & Application \\
\hline \multirow[t]{5}{*}{$\begin{array}{r}\text { Feedback } \\
\text { Control }\end{array}$} & {$[96]$} & Delayed FANN & $\begin{array}{l}\text { Riemann-Liouville } \\
\text { derivative }\end{array}$ & Synchronization \\
\hline & {$[43]$} & Nonidentical FANN & & Global synchronization \\
\hline & {$[178]$} & Memristive FANN & & $\begin{array}{l}\text { Projective } \\
\text { synchronization }\end{array}$ \\
\hline & {$[222]$} & $\begin{array}{l}\text { Complex-valued } \\
\text { memristive FANN }\end{array}$ & & $\begin{array}{l}\text { Global asymptotic } \\
\text { synchronization }\end{array}$ \\
\hline & {$[223]$} & $\begin{array}{l}\text { Delayed memristive } \\
\text { BAM FANN }\end{array}$ & & $\begin{array}{l}\text { Global projective } \\
\text { synchronization }\end{array}$ \\
\hline
\end{tabular}

Liouville derivatives are the only two derivatives used to reach stability in these systems.

Table 4 summarize research works related to ANN involved with FC to reach stability.

From the four tables shown in this manuscript: it's important to mention that temporal models with recurrent FANN have been developed in works: [41,45,79, $102,182,198,199,263,266]$.

\section{Other interesting applications of ANN involved with FC}

From the bibliographic analysis, there were found works where ANN was involved with FC to reach goals, such as approximation of functions, description of chaos, estimation, global dissipativity, periodicity, and modeling heat transfer process. Other researches have been applied to the different areas of science and engineering, such as medicine, image encryption, robotic, among others. The most important works related to these applications will be described in detail in this section, as follows.

\section{ANN involved with FC: applications in approximation of functions}

In several research works, there were approximated functions with the aid of ANN involved with FC. Some of these works will be explained in detail below:

The fractional quantitative approximation of realvalued functions involving Caputo derivative was carried out on feed-forward ANN in Ref. [286]. These approximations were derived by establishing Jackson type inequalities, converging the fractional approximation results better than the integer-order scheme.

The fractional differential polynomial ANN was the proposed method to approximate a multi-parametric function with polynomials, involving the Caputo derivative. The generalization depended on the RiemannLiouville differential operator, and the experimental results demonstrated that the approximation to the exact value with the fractional differential polynomial ANN was quicker than the integer-order method [287].
Liu and Fei in Ref. [165], approximated the nonlinear Dual Radial Basis functions ANN and the upper bound of estimated disturbances, improving the system stability and robustness, involving Caputo derivative. The ANNs weights were updated online to approximate the dual Radial Basis functions ANN structures, applied to a control system.

In Ref. [189], an adaptive control based on ANN was used to approximate unknown nonlinear functions using the fractional Lyapunov stability criterion and the backstepping technique, to control an uncertain fractional-order Chua-Hartley chaotic systems. The ANN was employed to approximate unknown system uncertainties and external disturbances. The numerical simulation was given to demonstrate the effectiveness of the proposed approach.

On the other hand, Lu and Wang in Ref. [288] developed the adaptive ANN tracking control using backstepping technology for the fractional-order chaotic permanent magnet synchronous motor with the immeasurable state, parameter uncertainties, and external load disturbance, involving Caputo derivative. The proposed approach employed a Chebyshev ANN and a state observer to approximate the unknown functions and estimate the unmeasurable state. In this work, the simulation results were presented to demonstrate the correctness of the proposed methodology.

In Ref. [289], authors developed an adaptive ANN control based on command filtered backstepping method for fractional-order permanent magnet synchronous motor with parameter uncertainties and unknown time delays, involving Caputo derivative. The unknown parameters, as well as the load disturbance, were approximated by using ANN. The time delays uncertainties were gotten by employing proper Lyapunov functions. Meanwhile, numerical simulations were given to demonstrate the effectiveness of the proposed method. Comparison among the proposed controller, classical backstepping controllers, and radial basis function ANN terminal sliding mode surface controller was carried out, showing the proposed controller to have a better performance.

Finally, fractional power series ANN for solving: delay fractional optimal control problems and fractional optimal control problems with equality and inequal- 


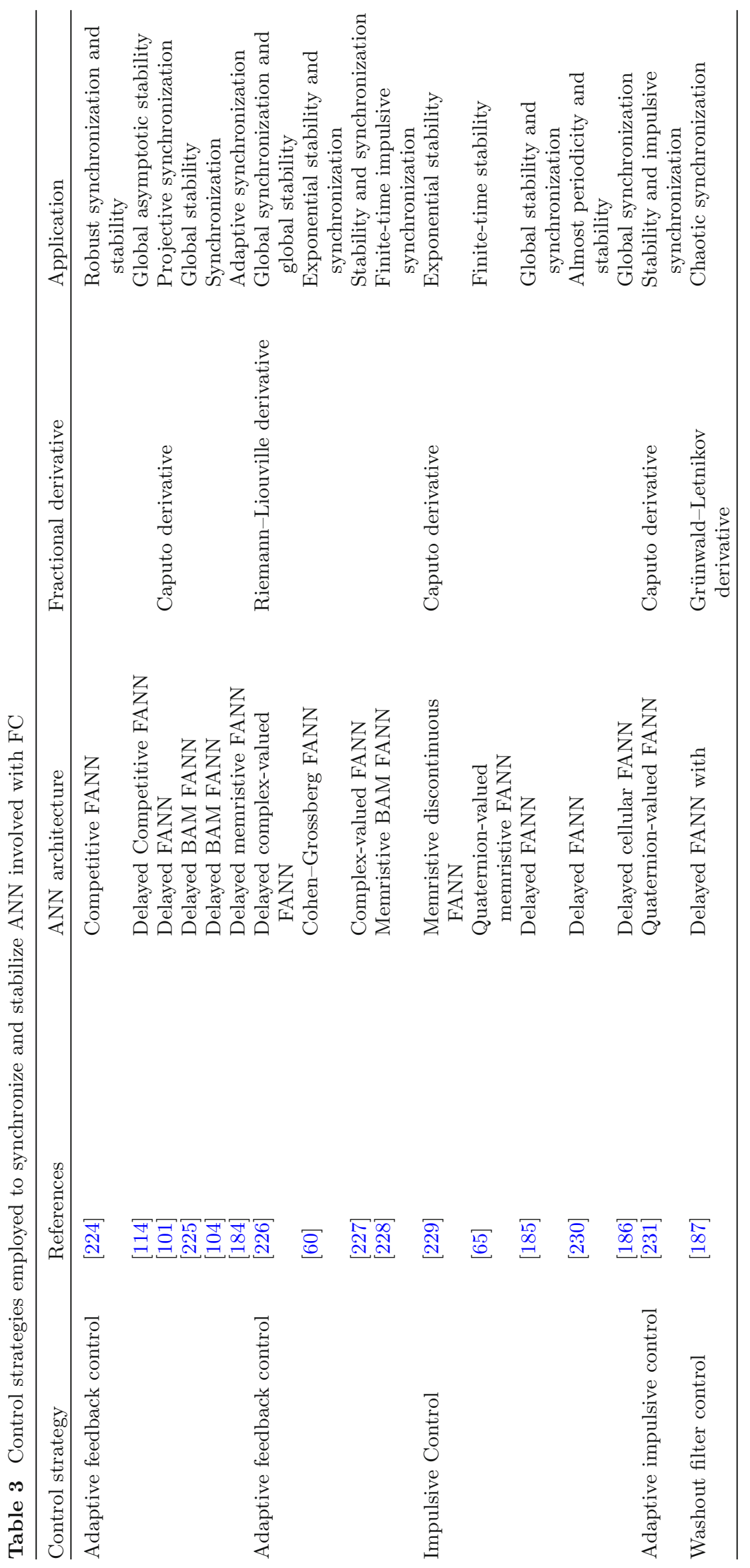


Table 4 ANN involved with FC to reach stability

\begin{tabular}{|c|c|c|c|}
\hline Application & References & ANN architecture & Fractional derivative \\
\hline \multirow[t]{4}{*}{ Exponential stability } & {$[171]$} & FANN & \\
\hline & {$[32]$} & Complex-valued FANN & Caputo derivative \\
\hline & {$[232]$} & Interval projection FANN & \\
\hline & {$[271]$} & Delayed BAM FANN & \\
\hline \multirow[t]{11}{*}{ Uniform stability } & {$[241]$} & FANN & \\
\hline & {$[237]$} & $\begin{array}{l}\text { FANN with and without } \\
\text { delays }\end{array}$ & \\
\hline & {$[76,242]$} & Delayed FANN & \\
\hline & {$[240]$} & Hopfield FANN & \\
\hline & {$[239]$} & Memristive FANN & \\
\hline & {$[272]$} & Delayed memristive FANN & Caputo derivative \\
\hline & {$[273]$} & $\begin{array}{l}\text { Delayed memristive fuzzy } \\
\text { BAM FANN }\end{array}$ & \\
\hline & {$[238]$} & Cellular FANN & \\
\hline & {$[79,233,234]$} & $\begin{array}{l}\text { Delayed complex-valued } \\
\text { FANN }\end{array}$ & \\
\hline & {$[235,236]$} & Delayed BAM FANN & \\
\hline & {$[274]$} & Delayed BAM FANN & \\
\hline \multirow[t]{11}{*}{ Finite-time Stability } & {$[248]$} & FANN & \\
\hline & {$[75,85,246,275]$} & Delayed FANN & \\
\hline & {$[247]$} & Delayed FANN & \\
\hline & {$[112,243,276]$} & $\begin{array}{l}\text { Delayed complex-valued } \\
\text { FANN }\end{array}$ & \\
\hline & {$[277]$} & $\begin{array}{l}\text { Delayed complex-valued } \\
\text { memristive FANN }\end{array}$ & \\
\hline & {$[244]$} & $\begin{array}{l}\text { Delayed complex-valued } \\
\text { memristive FANN }\end{array}$ & Caputo derivative \\
\hline & {$[175]$} & Cohen-Grossberg memristive & \\
\hline & {$[245]$} & $\begin{array}{l}\text { FANN } \\
\text { Delayed Cohen-Grossberg } \\
\text { FANN }\end{array}$ & \\
\hline & {$[89]$} & Delayed Hopfield FANN & \\
\hline & {$[207]$} & Delayed BAM FANN & \\
\hline & {$[103]$} & Delayed BAM FANN & \\
\hline \multirow[t]{11}{*}{ Global stability } & {$[28]$} & FANN & \\
\hline & {$[59]$} & BAM FANN & \\
\hline & {$[278]$} & Delayed BAM FANN & \\
\hline & {$[62]$} & Fuzzy FANN & \\
\hline & {$[38,256]$} & Hopfield FANN & \\
\hline & {$[86]$} & Delayed Hopfield FANN & Caputo derivative \\
\hline & {$[16]$} & Memristive FANN & \\
\hline & {$[117,174,180,279]$} & Delayed memristive FANN & \\
\hline & {$[106]$} & Delayed Cellular FANN & \\
\hline & {$[185,258]$} & Delayed cellular FANN & \\
\hline & {$[257]$} & $\begin{array}{l}\text { Delayed complex-valued } \\
\text { FANN }\end{array}$ & \\
\hline \multirow[t]{4}{*}{ Global Stability } & {$[259]$} & Delayed FANN & \\
\hline & {$[95]$} & Delayed FANN & Riemann-Liouville derivative \\
\hline & {$[280]$} & Delayed fuzzy BAM FANN & \\
\hline & {$[255]$} & Delayed hybrid BAM FANN & \\
\hline \multirow[t]{2}{*}{ Stability and Bifurcation } & {$[41]$} & Recurrent FANN & \\
\hline & {$[36,37]$} & Hopfield FANN & \\
\hline
\end{tabular}


Table 4 continued

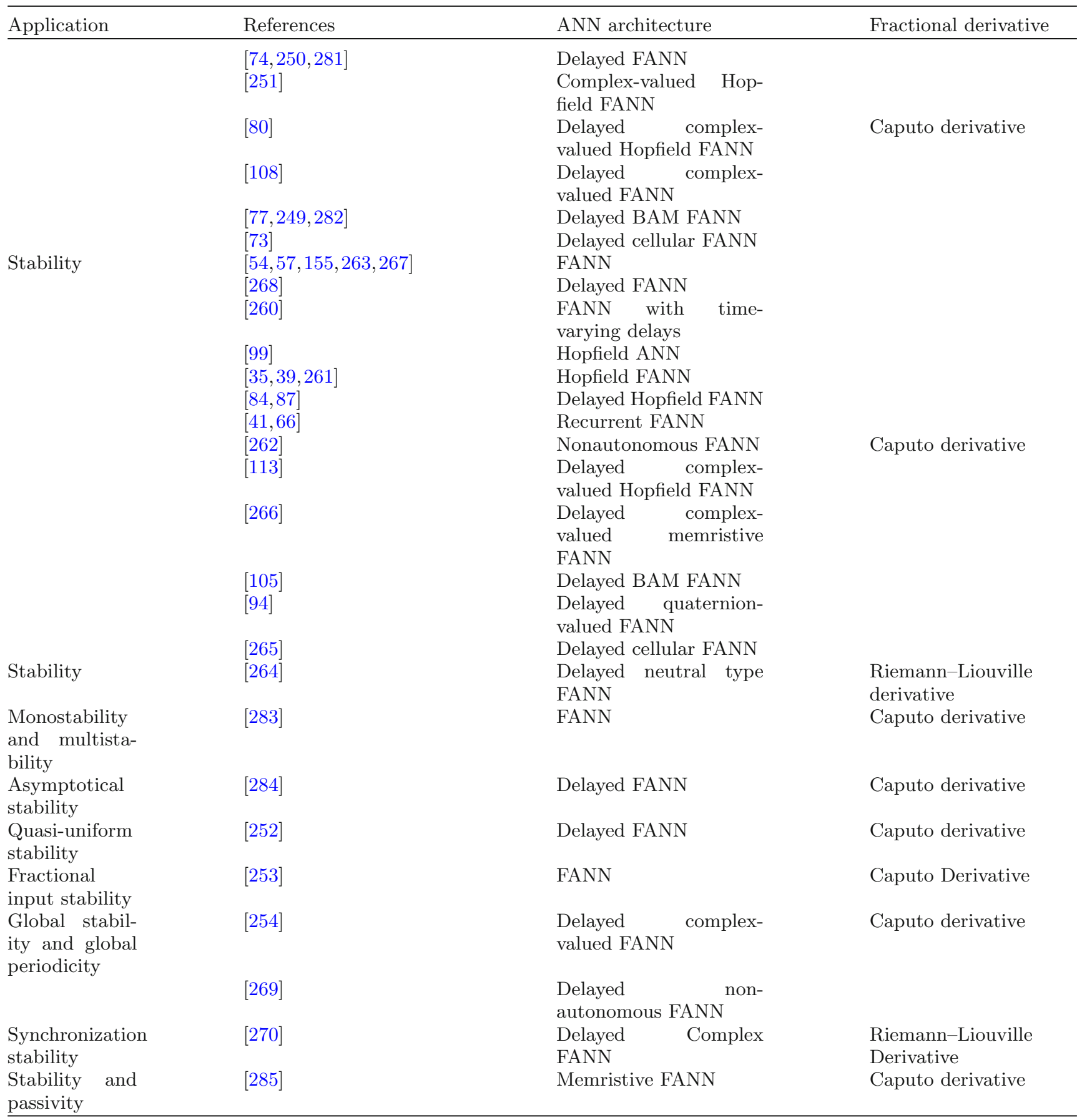

ity constraints were developed in $[47,69]$ respectively, involving Caputo derivative, feed-forward ANNs and according to the Pontryagin minimum principle. In both works, the optimization techniques and collocation methods were proposed to determine the approximate solution of the fractional optimal control problems. The obtained results were compared with the exact solutions and analytical solutions, respectively.

\section{ANN involved with FC: presence of chaos}

Next, several types of research where ANN involved with FC have chaotic behaviors will be depicted:

In Ref. [170], a numerical simulation algorithm for FDE was presented involving Riemann-Liouville derivative, where the chaotic phenomena and their control were discussed by numerical simulation. Chaos feedback control was developed, allowing to control and synchronize the FANN system. 
Moreover, in Ref. [290] obtained the fractional-order model of delayed cellular ANN for describing chaotic behaviors for fractional-order $0.1 \leq \alpha<1$ interval. Meanwhile, delay time values for which the chaos occurred were defined using the largest Lyapunov exponents. Riemann-Liouville derivative was employed in this work, and the simulation results demonstrated that the time delay where chaos occurred decreased as the fractional-order decreased too.

Moreover, Kaslik and Sivasundaram Seenith in Ref. [37] investigated the stability, multi-stability bifurcations, as well as the chaos of Hopfield FANN involving the Caputo derivative. In this work the critical values of fractional-order where the Hopf bifurcations occurred were identified, and the stability domain of a steadystate was characterized. The simulation results demonstrated that the chaotic behavior appeared when the fractional-order of the system increased.

In Refs. [31,83], the authors studied the behaviors of the complex dynamics of a cellular FANN and a delayed Hopfield FANN, respectively. To carried out these studies, numerical simulations involving the Caputo derivative were developed. The systems investigated in these works presented dynamic behaviors, such as periodic and chaotic motions. Furthermore, in both works, the existence of chaotic attractors was demonstrated. This was verified using bifurcation diagrams and phase portraits.

On the other hand, the projective synchronization of chaotic memristive FANN with time-varying delay and switching jumps mismatch involving RiemannLiouville derivative, was studied in [178]; also, in this work, the chaotic behavior of the memristor-based FANN system was showed.

Subsequently, Luo et al. in Ref. [156] developed an adaptive synchronization methodology combining Chebyshev ANN, extended state tracking differentiator, and adaptive backstepping, to reach the synchronization between the drive system and response system of a fractional-order chaotic arch micro-electro-mechanical system with the uncertain item and time delay under distributed electrostatic actuation. Caputo derivative was employed in this work, and the stability of the closed fractional-order arch micro-electromechanical system was guaranteed based on the fractional-order Lyapunov stability criterion. The simulation results demonstrated the effectiveness of the proposed scheme.

Moreover, in Ref. [291] stability, bifurcation, and chaos of a Memristive FANN with discontinuous memductance functions were investigated, employing Caputo derivative and identifying interesting dynamics, such as chaotic motion, tangent bifurcation, and intermittent chaos. The chaotic attractors were demonstrated to exist over a wide range of some specified parameters.

Otherwise, the chaotic Chua's attractor of fractionalorder was studied in Ref. [169]. In Ref. [292] the Lorenz system was studied, the chaotic attractors of Hopfield FANN in [293], memristor-based FANN in Ref. [210], two-dimensional delayed FANN in Ref. [101], timedelayed inertial FANN in Ref. [220], and nonidentical FANN chaotic behaviors were studied in Ref. [163].
Finally, Han in Ref. [200] performed a composite learning sliding mode control approach to attain the synchronization of chaotic FANN with unmatched unknown parameters, employing Caputo definition. A comparison between the proposed composite learning sliding mode control and the common sliding mode control demonstrated that the proposed composite learning sliding mode control establishes an accurate parameter estimation without the permanent excitation condition having better control performance than the sliding mode control scheme.

Other research related to chaos of ANN involved with FC applied to image encryption will be found later in this paper, [82,186,294] and [295].

\section{ANN involved with FC: applications in estimation}

The main works about estimation involving ANN and FC will be depicted in the following summary:

On-line state estimation of nonlinear dynamic systems was carried out using Differential FANN. The simulation of two coupled tanks was carried out to demonstrate the feasibility of Differential FANN as a nonlinear systems identifier [296]. In [35], the same author solved a parameter estimation problem for demonstrating the Hopfield FAN existence using the Caputo derivative. In this case, the stability of Hopfield FANN was reached applying an energy-like function analysis.

Besides, in Ref. [11] authors studied the parameter estimation problem of unknown system parameters on FANN involving Caputo and Riemann-Liouville derivatives. Synchronization-based identification method of fractional-order was achieved thanks to the combination of adaptive control and parameter update law, demonstrating the correctness of the obtained results through a numerical example.

Moreover, in Refs. [297,298] were designed a state estimator and non-fragile state estimator for delayed memristive FANN involving the Caputo derivative, respectively. In work [297], the state estimators' existence was ensured, and a suitable state estimator for memristive FANN was proposed. Accordingly, based on the fractional-order Lyapunov direct method, some new sufficient conditions were given to guarantee the existence of the state estimator. On the other hand, in Ref. [298] by using the Lyapunov technique, the authors were getting sufficient conditions to ensure the global asymptotic stability of the error model.

A radial basis function ANN was used to estimate the bound of disturbances in Ref. [299]. And an adaptive fractional sliding mode controller with a neural estimator for a class of nonlinear systems also was designed.

A robust control law was designed to guarantee the occurrence of the sliding motion, as well as the Hopfield FANN stabilization involving the RiemannLiouville derivative in Ref. [164]. Moreover, the system's unknown parameters were estimated, and the sliding surface to origin finite-time stability was achieved based on the fractional-order Lyapunov theory. An example of 
Hopfield FANN was presented to demonstrate the effectiveness of the proposed scheme.

The non-fragile state estimation issue for memristive BAM FANN with and without time delays was studied in Ref. [300] by applying the fractional-order derivative in Caputo sense. Based on fractional-order Lyapunov functionals and linear matrix inequalities was ensured the asymptotical stability of the error system.

The quasi-estimation was investigated by $\mathrm{Li}$ in Ref. [301] employing fuzzy memristive FANN based on the Caputo derivative. The quasi-estimation was studied through a Laplace transformation, and the quasisynchronization control was attained due to the designed feedback controller.

Besides, in Ref. [104], the authors studied the MittagLeffler state estimator and an adaptive synchronization for delayed BAM FANN by applying the Caputo derivative. An adaptive feedback control was designed, and Mittag-Leffler adaptive synchronization was reached using fractional-order inequality techniques.

Another novel approach in this area is the robust state estimation of complex-valued FANN with uncertain parameters and BAM FANN with norm-bounded uncertainties, investigated in Refs. [278,302], respectively. Hu in Ref. [302], applied the Riemann-Liouville derivative, and Nagamani in [278] used the Caputo derivative. Since both systems presented time delays, a new linear matrix inequality criterion was obtained to reach the asymptotic stability of the systems' error. In both cases, numerical simulations were performed to confirm the effectiveness of the proposed schemes.

Finally, in Refs. [120,303], the authors investigated the finite-time projective synchronization of memristive FANN with mixed time-varying delays and uncertain parameters and the finite-time synchronization of memristive Cohen-Grossberg FANN with time-varying delays, respectively. In these works, the Caputo derivative and feedback controllers were applied. Moreover, the settling times were estimated.

\section{ANN involved with FC: applications in dissipativity}

Following relevant works related to dissipativity involving FC applied to ANN will be shown:

First, in Ref. [100], the authors investigated the global dissipativity of delayed FANN and discontinuous activation functions employing Caputo derivative. In this research, sufficient conditions were given to ensure the dissipativity of the model solution. The effectiveness of the proposed scheme was demonstrated by numerical examples.

Second, the dissipativity and global asymptotic stability of delayed complex-valued FANN were investigated in Ref. [257]. In this research, the authors used the Caputo derivative. Numerical simulations showed the effectiveness of the proposed scheme.

Finally, Li in Ref. [304] investigated the dissipativity and the exponential synchronization control of Memristive FANN involving reaction-diffusion terms, the Caputo derivative, and a feedback controller. The proposed scheme results presented fewer conservation effects when comparing with other works found in the literature.

\section{ANN involved with FC: applications in periodicity}

The overview of the research works focused on the periodicity involving FC on ANN will be depicted below:

First, in Ref. [71], the authors demonstrated that the fractional derivative of a periodic function cannot be a periodic function with the same period involving Caputo, Riemann-Liouville, and Grünwald-Letnikov derivatives; in this paper a FANN was employed to guarantee the non-existence of periodic solutions in fractional-order dynamical systems.

Subsequently, Wu and Zeng in Ref. [62] derived the S-asymptotic $\omega$-periodicity and global asymptotic $\omega$ periodicity of fuzzy FANN, involving Caputo derivative. The difference between integer-order neurodynamic systems and fractional-order neurodynamic systems was shown. Several simulations were performed to demonstrate the effectiveness of the proposed method.

On the other hand, the global stability and global asymptotic periodicity for complex-valued FANN with time-varying delays was discussed in Ref. [254], while for non-autonomous FANN with time-varying delays was addressed in Ref. [269] by applying the Caputo derivative. In these works, the solutions converged to the same periodic function. Numerical examples were given to demonstrate the feasibility of the schemes.

Finally, the global asymptotic $\omega$-periodicity for a non-autonomous FANN involving Caputo derivative was investigated in [262]. The authors demonstrated that FANN has S-asymptotically periodic solutions. Furthermore, all solutions of FANN globally converge to a periodic function.

\section{Heat transfer process}

Only one work related to the FC on ANN with the heat transfer process will be depicted as follows:

The heat transfer process was modeled using a discrete FANN involving Grünwald-Letnikov derivative in Ref. [305]. The experiments and the obtained results showed that the proposed FANN modeled the unknown dynamics correctly.

\section{ANN involved with FC: applications in sustainable energy}

ANN involved with FC has been applied in the sustainable energy area, specifically in wind turbine applications $[30,34,149]$. These works will be addressed below:

The behavior of a wind turbine in operation was identified using a variable order FANN and a Dynamic FANN involving the Caputo derivative in Refs. [30,34], respectively. The proposed methods were evaluated and validated by using experimental data obtained from the wind turbine under operation. Moreover, in Ref. [34], results obtained with the dynamic FANN were com- 
pared with the obtained with the integer-order dynamic ANN showing that the fractional approach was more accurate.

Also, in Ref. [149] was presented a fractional-order PID control implementation for regulating both the rotor speed and the power production of a wind turbine. The control scheme was combined with a radial basis function ANN, allowing it to reach better performance and robustness than with the integer-order controllers.

\section{ANN involved with FC: applications in medicine}

ANN involved with FC has been applied to the medical field and reported in Refs. $[24,145,197,306]$. Below we will describe these research works.

In Ref. [145], the detection of left-sided and rightsided hearing loss was carried out using the fractional Fourier transform and a feedforward ANN trained by the Levenberg-Marquardt algorithm. Also, in Ref. [306] the authors developed a pathological brain detection system based on multi-layer perceptron ANN to improve the interpretation of magnetic resonance brain images. In this work, the ANN was the classifier that received the fractional Fourier entropy features extracted from the brain images. The adaptive realcoded biogeography-based optimization was the algorithm implemented to train this ANN, and the proposed method was able to interpret the images with an accuracy of $99.53 \%$, improving the results obtained from other pathological brain detection system.

Subsequently, Sharafian in Ref. [197] used a radial basis function ANN with a sliding mode observer for modeling the uncertainties of the human immunodeficiency virus infection fractional model. The fractional mathematical model involved the Caputo derivative. The ANN estimated the system uncertainties while the sliding mode observer eliminated the external disturbances. In this work, the finite-time stability of the observer was guaranteed. In fact, the radial basis function ANN estimated the complex nonlinearity of the system accurately.

Finally, in Ref. [24], the fractional-order Darwinian particle swarm optimization (FO-DPSO) algorithm was employed for training a feed-forward ANN to approximate the solutions of the corneal shape model for eye surgery. In this work, the Adams-Bashforth-Moulton numerical method was used to show the effectiveness of the proposed scheme

\section{ANN involved with FC: applications in unmanned aerial vehicles (UAVs)}

ANN involved with FC has been applied to unmanned aerial vehicles (UAVs). Some relevant works have been reported in Refs. [144,307]. Following, we will address these works.

In Ref. [144], the authors approximated the realization of an analogical fractional-order PID controller using feed-forward ANN and a finite impulse response filter to establish a robust control for applications of unmanned aerial vehicles (UAVs). Posteriorly, in Mobarez et al. the authors implemented a fractionalorder PID control based on ANN for fixed-wing UAVs, where the proposed autopilot was evaluated in linearized and nonlinear systems. The fractional controller showed better performance against wind disturbance, the effect of the sensors' noise, and system uncertainties when compared with other controllers.

\section{ANN involved with FC: applications in circuits realization}

ANN involved with FC has been applied to circuit realization. In works $[25,165,189,198,199,292]$ relevant research have been presented on this issue. Below we will present an overview of these works:

In Ref. [165], the authors developed an adaptive fractional sliding mode control involving the Caputo derivative. The fractional controller was based on the dual radial basis function ANN, allowing to improve the performance of three-phase shunt active power filters. Also, the Chua's circuit with Caputo derivative was developed in Ref. [25] to investigating the fractional Chua's system and discuss its chaotic behavior. Furthermore, in Ref. [189], the authors developed an adaptive ANN backstepping control of fractional-order Chua-Hartley chaotic system, as well as an electronic circuit. In this work, the ANN was used to estimate the unknown nonlinear function, and the proposed controller was able to guarantee the stability of the closed-loop system. Otherwise, in Ref. [292], a lathe machine tool was the basis to study turning chatter vibration by means of ANNbased on Chua's circuit and fractional-order Lorenz master/slave chaotic system. Finally, fractional-order sliding mode controllers based on recurrent ANN were developed for the current compensation and the current harmonic compensation of active power filter involving Caputo derivative in $[198,199]$, respectively.

Also, Liu [308] employed a reaction/diffusion cellular FANN to describe the diffusion behavior that happened on the electromagnetic field where the electrons describe nonuniform movement. In this work, the Caputo derivative was used, and the stability of the FANN has achieved thanks to an observed-based boundary control.

On the other hand, Ding et al. [309] accomplished the first sampling-controlled memristive FANN with stochastic sensor faults via an impulsive method based on Caputo derivative. This novel approach was applied to the fractional-order Chua's circuit system, where they reached its stabilization.

Finally, Sanchez et al. [310] simulated the incremental capacity curve of an LFP battery model by training a recurrent FANN. In this work, the model-based health prognosis of the LFP battery with an accuracy that is comparable with the laboratory measurements was attained. 


\section{ANN involved with FC: applications in robotic}

ANN involved with FC and Models to control Robots have been developed in Refs. [52,68], and they will be summarized as follows:

In Ref. [52], the authors proposed a deep convolutional ANN based on the fractional-order sliding mode control scheme to control trajectory tracking of robotic manipulators involving Caputo derivative. The proposed control showed to have robust performance against parametric uncertainties and external disturbances. In this work, several simulations were carried out to validate the proposed methods.

In Ref. [68] was presented a nonlinear neural fractional-order PID controller based on ANN applied to the motion control of nonholonomic differential drive mobile robot, involving Grünwald-Letnikov derivative. The hybridization of a modified adaptive PSO and the EFF optimization algorithms were implemented for tuning the parameters of the fractional-order PID controller based on ANN. The authors showed with numerical simulations that the performance of the designed fractional controller was excellent compared with nonlinear controllers of integer-order on the trajectory tracking of the differential drive mobile robot with different trajectories as study cases.

\section{ANN involved with FC: applications in image encryption}

ANN involved with FC have been applied to image encryption in Refs.: [82,186,294,295]. Such works will be overviewed as follows:

First, in Ref. [186], the authors presented an image encryption approach based on impulsive synchronization of chaotic ANN applying the fractional-order approach involving Caputo derivative and considering a delayed cellular FANN.

Second, image encryption algorithms were designed based on chaotic fuzzy cellular neural FANN with timevarying delays and chaotic three-dimensional discrete Hopfield FANN in Refs. [82,294], respectively. The authors used the Caputo derivative and the FANNs as a pseudo-random number generator. The dynamic behavior and synchronization of the systems were investigated and applied to image encryption algorithms where solutions allowed to improve encryption security. Third, in [82], numerical evaluations were developed, and analysis of bifurcation diagrams, phase space diagrams, and time series plots to explore the effects of the time-varying delay and the fractional-order. In this work, the global stability conditions were derived under the design of an adaptive control approach guaranteeing the global asymptotic and the exponential stability by synchronizing the drive-response system when time tends to large. On the other hand, in Ref. [294], phase portraits, bifurcation diagrams, and Lyapunov exponents were developed to show the chaotic dynamics of the system. A control approach was designed to synchronize the system. The results show the effective- ness of the encryption system. The simulation results demonstrated that the algorithms have good encryption features.

Similar to the research works presented above, in Ref. [295], another chaotic image encryption algorithm was proposed. In this case, a five-dimensional cellular FANN was employed as a diffusion controller in the encryption system. The results showed that this new algorithm improves encryption efficiency with good security performance. Furthermore, it resisted the common attack methods.

Finally, the synchronization of a class of FANNs was carried out by designing an adaptive control to develop a crypto-system algorithm for encryption/decryption of unmanned aerial vehicle color images in secure communications, employing Caputo derivative [311].

\section{ANN involved with FC: applications in other engineering applications}

Blasik et al. [312] developed an accurate numerical method based on ANN and Caputo derivative to extend the front fixing method developed in a previous work based on the one phase fractional-order Stefan problem (anomalous molecular diffusion where the diffusion coefficient is generalized).

\section{Future research topics arising on ANNs involved with FC}

The main advantage of modeling systems involving FANNs is that the fractional derivatives are an excellent tool in describing the memory and hereditary properties of various processes. Therefore, the simulated systems involving FANNs are more accurate than the integerorder models. However, there are few works done in this field to date, and there are many important topics that have not been approached yet. Therefore, we would like to recommend some future research topics where the FANNs could be successfully applied, which are detailed as follows:

- We propose to apply the FANNs to the identification, control, performance studies, and prediction of behaviors on physical models. Thus, the models could improve their accuracy without increasing their complexity.

- The employment of FANNS for the simulation of trajectorial physical models could allow that the effectiveness of the trajectories prediction to be increased, avoiding wasting time and resources in the experimental studies.

- The management of the plants of industrial processes is usually complex in nature. Often, the training ANN models must attain a fast response concerning corresponding physical models and for the real-time monitoring of the plants; We propose to use FANNs to model the indus- 
trial plants since FANNs could do the management of them more efficiently with the fractional approach on their simulations.

- We propose to apply the FANNs on the sustainable energy technologies to realize a better analysis and optimization of their parameter, and thus, help environmental care.

- FANNs could be an accurate tool for the study of behaviors, the prediction of performance, and the optimization of solar collectors, biomass heating systems, wind turbines, and so on.

- FANNs could be an excellent tool in studying voltage prediction on PEM fuel cells with minimum time demand and good accuracy, reducing costs and avoiding extensive experiments. FANNs could lead to a better analysis of the PEM fuel cell components and better optimize their parameters to minimize the voltage losses and reach their best efficiency.

- We propose to employ FANNs on modeling of the epidemic spreading of viruses that affect the health of animals and human beings.

- The employment of FANNs in modeling the parameters that determine the diagnosis of COVID-19 would provide fast and accurate diagnostics of the actual COVID-19 pandemic, helping clinicians in detecting COVID-19, quantification, follow-up of the infected cases, and helping the activation of the plan actions.

- The spreading prediction and analysis of the performance of common viruses such as HIV, H1N1, Dengue, Ebola, and others could be successfully modeled using FANNs, which is beneficial for preserving lives since predictions are helpful to control and prevent the spread of the viruses.

- We propose to employ FANNs to model the spread of cancer cells from where they first formed to another part of the body.

- The identification of cancer cells and the prediction of their movement into the human body are essential for preserving life. Thanks to the effectiveness of FANNs on modeling and predicting behaviors in different areas of science, we believe that some accurate FANNs models based on cancer cell propagation could help in its identification and prediction.

- We propose to apply the FANNs for the simulation of biological processes.

- FANNs could be efficiently used in the process control of biological systems, reaching a better online optimization of these systems than in the integer-order cases.

- We propose to simulate probabilistic models using FANNs.

- The meteorological time series prediction could be more accurate when the ANN describes the system as simulated employing FC. Guaranteeing a good prediction, the forecast of human and economic losses in front of natural disasters could be employed successfully.

- We propose combining fractional-order physicallybased modeling and deep learning.

- The fractional deep neural networks are a tool of machine learning and artificial intelligence more powerful than integer-order deep neural networks; the FC improves the deep learning methods, reaching a better optimization of parameters of the physical models.

- We propose the application of FANNs to predict economic trends.

- The realization of economic models based on FANNs could help economists and business people accurately identify entrepreneurship opportunities. This approach could help governments and economists establish correct predictions of the prices of crude oil, natural gas, power, risk management, trading strategies, etc.

\section{Conclusions}

This manuscript presented a bibliographic review of fractional calculus (FC) on artificial neural networks (ANN). We have focussed on realizing a thorough investigation related to the employment of $\mathrm{FC}$ on $\mathrm{ANN}$, the methods used to solve their fractional differential equations (FDE), the optimization algorithms employed to train these systems, the control approaches involved with them, and their main applications in different areas of science and engineering. According to the bibliographic review, the most used method to solve the FDE on ANNE involved with FC is the analytical Laplace transform method, followed by the AdamsBashforth-Moulton method. Also, other methods have been used to solve FDE in these systems, such as the Homotopy perturbation method, the power series expansion method, Adomian decomposition method, among others. All of them have allowed establishing a comparison between the proposed schemes and the analytical or numerical solution of FDE to validate them. Regarding the optimization algorithms used for the training of ANN involved with FC, there have been developed a few algorithms with the fractional approach, indeed, just gradient descent algorithm, Back-propagation algorithm, and Darwinian particle swarm optimization algorithm have their fractional version. Nevertheless, there are lots of classical algorithms employed with the aim of training ANN involved with FC. According to the advantages of FC, most of these algorithms could be implemented with a fractional approach in the future, obtaining results very different from the obtained using classical algorithms and probably with solutions more near reality. For training of these systems, the most used derivative was the 
Caputo derivative, followed by the Riemann-Liouville derivative, Atangana-Baleanu in Caputo's sense, and Grünwald-Letnikov, respectively. Otherwise, another important topic that has been presented here is the control of such systems; since several works joined control strategies with FC to guarantee synchronization and stabilization of the ANN involved with FC. Concerning the control strategies, the most reported in the literature is the feedback control, followed by adaptive control, sliding mode control, adaptive feedback control, impulsive control, and washout filter control. Therefore, we can affirm that FC is an excellent mathematical tool to be used with ANN. Also, we have confirmed that the Caputo derivative is the most applied with ANN due to its ability to describe physical systems. The RiemannLiouville derivative is the second derivative most used on these types of systems. This derivative is applied to describe theoretical systems, while the GrünwaldLetnikov derivative is less used in these types of systems. Other interesting applications of ANN involved with FC are the approximation of functions, the dissipativity, periodicity demonstration, description of chaotic behaviors, among others. But some of the most important advances related to ANN involved with FC are their recent applications in medicine, robotic, cryptography, image processing, and sustainable energy. This implies that the research community is paying attention to the fractional calculus theory.

We are in the presence of an interesting branch of mathematics compared with integer-order calculus. The FC can better describe several processes' memory and genetic characteristics, having unlimited memory and more degrees of freedom.

However, few applications exist until today; we consider many promising works to do shortly. Therefore, we decided to guide the researchers for the realization of future works in this field. Some of the future directions we suggested are the following: the application of FANNs on the study of trajectorial physical models for reach more accurate simulated systems and better prediction of trajectories than in integer-order cases; the employment of FANNs for the improvement of the voltage prediction in PEM fuel cells and better identification of their parameters; the simulation of industrial plants using FANNs to reach more accurate computational systems which can be managed on real-time with fast-response. In medicine and biology, we proposed implementing FANNs based on the epidemic spreading of viruses such as Covid-19, HIV, Ebola, H1N1, and others for a better analysis, diagnosis, prediction, and forecast of them. Also, we proposed to realize the simulation of cancer cells spreading into the human body to help clinicians detect, study the behavior of cancer cells, and predict their performance accurately, helping save lives. Besides, we proposed implementing FANNs on the biology process to aid the development of process control of biological systems and predict the performance of these systems, avoiding realize exhaustive failed experiments. On the other hand, we recommended applying FANNs on probabilistic meteorological models to attain accurate systems capable of making good predictions avoiding human and economic losses when natural disasters happen. Finally, but not less important, we recommended implementing the FANNs based on economic models for guiding governments and economists to make decisions based on a correct prediction of the economic phenomena. All our recommendations are based on the certainty that the accurate models reached employing FANNs avoid the employment of exhaustive repetitive experimental tests. Thus, the FANNs help the growth of science and engineering less time employing the optimum resources for their development.

Acknowledgements Edumis Viera Martin acknowledge the support provided by CONACyT through the assignment doctoral fellowship. Jesús Emmanuel Solís Pérez acknowledges the support provided by CONACyT through the assignment post-doctoral fellowship. José Francisco Gómez Aguilar acknowledges the support provided by CONACyT: cátedras CONACyT para jóvenes investigadores 2014. José Francisco Gómez Aguilar, Jesús Emmanuel Solís Pérez, José Alfredo Hernández-Pérez and Ricardo Fabricio Escobar-Jiménez acknowledges the support provided by SNI-CONACyT.

\section{Declarations}

Conflict of interest The authors declare no conflict of interest.

\section{References}

1. Z. Wang, X. Wang, Y. Li, X. Huang, Stability and Hopf bifurcation of fractional-order complex-valued single neuron model with time delay. Int. J. Bifurc. Chaos 27(13), 1750209 (2018)

2. R. Rakkiyappan, G. Velmurugan, F.A. Rihan, S. Lakshmanan, Stability analysis of memristor-based complex-valued recurrent neural networks with time delays. Complexity 21(4), 14-39 (2016)

3. C.J. Zúñiga-Aguilar, H.M. Romero-Ugalde, J.F. Gómez-Aguilar, R.F. Escobar-Jiménez, M. ValtierraRodríguez, Solving fractional differential equations of variable-order involving operators with Mittag-Leffler kernel using artificial neural networks. Chaos Solitons Fract. 103, 382-403 (2017)

4. S. Qureshi, A. Yusuf, A. Shaikh, M. Inc, Transmission dynamics of varicella zoster virus modeled by classical and novel fractional operators using real statistical data. Phys. A 534, 1-12 (2019)

5. A. Jajarmi, A. Yusuf, D. Baleanu, M. Inc, A new fractional HRSV model and its optimal control: a nonsingular operator approach. Phys. A 547, 1-12 (2020)

6. S. Qureshi, A. Yusuf, A. Ali Shaikh, M. Inc, D. Baleanu, Mathematical modeling for adsorption process of dye removal nonlinear equation using power law and exponentially decaying kernels. Chaos Interdiscip. J. Nonlinear Sci. 30, 1-10 (2020) 
7. A. Khan, G. Hussain, M. Inc, G. Zaman, Existence, uniqueness, and stability of fractional hepatitis B epidemic model. Chaos Interdiscip. J. Nonlinear Sci. 30, 1-10 (2020)

8. M. Inc, N. Bouteraa, M. Akinlar, S. Benaicha, Y. Chu, G. Weber, B. Almohsen, New positive solutions of nonlinear elliptic PDEs. Appl. Sci. 10, 1-13 (2020)

9. A. Yusuf, B. Acay, U. Mustapha, M. Inc, D. Baleanu, Mathematical modeling of pine wilt disease with Caputo fractional operator. Chaos Solitons Fract. 143, 1-13 (2021)

10. B. Acay, M. Inc, Fractional modeling of temperature dynamics of a building with singular kernels. Chaos Solitons Fract. 142, 1-11 (2021)

11. Y. Gu, Y. Yu, H. Wang, Synchronization-based parameter estimation of fractional-order neural networks. Phys. A 483, 351-361 (2017). https://doi.org/10.1016/ j.physa.2017.04.124

12. M. Asif, Z. Raja, R. Samar, M. Anwar, Design of unsupervised fractional neural network model optimized with interior point algorithm for solving Bagley-Torvik equation. Math. Comput. Simul. 132, 139-158 (2017). https://doi.org/10.1016/j.matcom.2016.08.002

13. J. Yu, C. Hu, H. Jiang, X. Fan, Projective synchronization for fractional neural networks. Neural Netw. 49, 87-95 (2014). https://doi.org/10.1016/j.neunet.2013. 10.002

14. L.M. Wang, Q.K. Song, Z.J. Zhao, Global asymptotic stability of memristor-based fractional-order complexvalued neural networks with time delays. Appl. Math. Mech. 38(3), 333-346 (2017). https://doi.org/10.1016/ j.neucom.2017.02.086

15. Z. Ding, Y. Shen, Projective synchronization of nonidentical fractional-order neural networks based on sliding mode controller. Neural Netw. 76, 97-105 (2016). https://doi.org/10.1016/j.neunet.2016.01.006

16. A. Wu, Z. Zeng, Global Mittag-Leffler stabilization of fractional-order memristive neural networks. IEEE Trans. Neural Netw. Learn. Syst. 28(1), 206-217 (2017)

17. I. Podlubny, in Fractional Differential Equations: An Introduction to Fractional Derivatives, Fractional Differential Equations, to Methods of Their Solution and Some of Their Applications, vol. 198 (Elsevier, 1998)

18. A. Atangana, D. Baleanu, New fractional derivatives with nonlocal and non-singular kernel: theory and application to heat transfer model. Therm. Sci. 20, 763-769 (2016)

19. K. Rajagopal, M. Tuna, A. Karthikeyan, s Koyuncu, P. Duraisamy, A. Akgul, Dynamical analysis, sliding mode synchronization of a fractional-order memristor Hopfield neural network with parameter uncertainties and its non-fractional-order FPGA implementation. Eur. Phys. J. Spec. Top. 228(10), 2065-2080 (2019)

20. X. Yang, C. Li, Q. Song, J. Chen, J. Huang, Global Mittag-Leffler stability and synchronization analysis of fractional-order quaternion-valued neural networks with linear threshold neurons. Neural Netw. 105, 88103 (2018). https://doi.org/10.1016/j.neunet.2018.04. 015

21. A.H. Hadian-Rasanan, D. Rahmati, S. Gorgin, K. Parand, A single layer fractional orthogonal neural network for solving various types of Lane-Emden equa- tion. New Astron. 75(2019), 101307 (2020). https:// doi.org/10.1016/j.newast.2019.101307

22. A.H. HadianRasanan, N. Bajalan, K. Parand, J.A. Rad, Simulation of nonlinear fractional dynamics arising in the modeling of cognitive decision making using a new fractional neural network. Math. Methods Appl. Sci. 43(3), 1437-1466 (2020)

23. M. Syed Ali, G. Narayanan, V. Shekher, A. Alsaedi, B. Ahmad, Global Mittag-Leffler stability analysis of impulsive fractional-order complex-valued BAM neural networks with time varying delays. Commun. Nonlinear Sci. Numer. Simul. 83, 105088 (2020). https://doi. org/10.1016/j.cnsns.2019.105088

24. W. Waseem, M. Sulaiman, A. Alhindi, H. Alhakami, A soft computing approach based on fractional order DPSO algorithm designed to solve the corneal model for eye surgery. IEEE Access 8(c), 61576-61592 (2020)

25. N.A. Khan, T. Hameed, O.A. Razzaq, M. Ayaz, Tracking the chaotic behaviour of fractional-order Chua's system by Mexican hat wavelet-based artificial neural network. J. Low Freq. Noise Vib. Act. Control 38(3-4), 1279-1296 (2018)

26. M. Pakdaman, A. Ahmadian, S. Effati, S. Salahshour, D. Baleanu, Solving differential equations of fractional order using an optimization technique based on training artificial neural network. Appl. Math. Comput. 293, 81-95 (2017). https://doi.org/10.1016/j.amc. 2016.07.021

27. Jing, Y., Dong, H., Liang, G.: Study on characteristic of fractional master-slave neural network, in Proceedings-2012 5th International Symposium on Computational Intelligence and Design, ISCID 2012, vol. 2. (IEEE, 2012), pp. 498-501

28. S. Zhang, Y. Yu, J. Yu, LMI conditions for global stability of fractional-order neural networks. IEEE Trans. Neural Netw. Learn. Syst. 28(10), 2423-2433 (2017)

29. Z. Yang, J. Li, X. Tang, Y. Niu, Hybrid projective synchronization of fractional-order neural networks with different dimensions. J. Phys. Soc. Jpn. 88(8), 1-6 (2019)

30. Z. Aslipour, A. Yazdizadeh, Identification of nonlinear systems using adaptive variable-order fractional neural networks (case study: a wind turbine with practical results). Eng. Appl. Artif. Intell. 85(2018), 462473 (2019). https://doi.org/10.1016/j.engappai.2019. 06.025

31. X. Huang, Z. Zhao, Z. Wang, Y. Li, Chaos and hyperchaos in fractional-order cellular neural networks. Neurocomputing 94, 13-21 (2012). https://doi.org/10. 1016/j.neucom.2012.01.011

32. J. Jian, P. Wan, Lagrange $\alpha$-exponential stability and $\alpha$-exponential convergence for fractional-order complex-valued neural networks. Neural Netw. 91, 1-10 (2017). https://doi.org/10.1016/j.neunet.2017.03. 011

33. Z. Aslipour, A. Yazdizadeh, Identification of Damavand tokamak using fractional order dynamic neural network. Trans. Inst. Meas. Control. 41(5), 1447-1457 (2019)

34. Aslipour, Yazdizadeh, Identification of wind turbine using fractional order dynamic neural network and optimization algorithm. Int. J. Eng. 33(2), 277-284 (2020) 
35. A. Boroomand, M.B. Menhaj, Fractional-order Hopfield neural networks, in Lecture Notes in Computer Science (including subseries Lecture Notes in Artificial Intelligence and Lecture Notes in Bioinformatics), vol. 5506 LNCS, no. PART 1 (2009), pp. 883-890

36. E. Kaslik, S. Sivasundaram, Dynamics of fractionalorder neural networks, in The 2011 International Joint Conference on Neural Networks. (IEEE, 2011), pp. 611-618

37. E. Kaslik, S. Sivasundaram Seenith, Nonlinear dynamics and chaos in fractional-order neural networks. Neural Netw. 32, 245-256 (2012). https://doi.org/10.1016/ j.neunet.2012.02.030

38. S. Zhang, Y. Yu, W. Hu, Robust stability analysis of fractional-order Hopfield neural networks with parameter uncertainties. Math. Probl. Eng. 2014, 1-22 (2014)

39. C.A. Tavares, T.M. Santos, N.H. Lemes, J.P. dos Santos, J.C. Ferreira, J.P. Braga, Solving ill-posed problems faster using fractional-order Hopfield neural network. J. Comput. Appl. Math. 381, 112984 (2020). https://doi.org/10.1016/j.cam.2020.112984

40. J. Jia, Z. Zeng, LMI-based criterion for global MittagLeffler lag quasi-synchronization of fractional-order memristor-based neural networks via linear feedback pinning control. Neurocomputing 412, 226-243 (2020). https://doi.org/10.1016/j.neucom.2020.05.074

41. M. Xiao, W.X. Zheng, G. Jiang, J. Cao, Undamped oscillations generated by Hopf bifurcations in fractional-order recurrent neural networks with Caputo derivative. IEEE Trans. Neural Netw. Learn. Syst. 26(12), 3201-3214 (2015)

42. M.A.Z. Raja, I.M. Qureshi, J.A. Khan, Swarm intelligence optimized neural networks for solving fractional differential equations. Int. J. Innov. Comput. Inf. Control 7(11), 6301-6318 (2011)

43. T. Hu, X. Zhang, S. Zhong, Global asymptotic synchronization of nonidentical fractional-order neural networks. Neurocomputing 313, 39-46 (2018). https:// doi.org/10.1016/j.neucom.2018.05.098

44. M.A.Z. Raja, M.A. Manzar, R. Samar, An efficient computational intelligence approach for solving fractional order Riccati equations using ANN and SQP. Appl. Math. Model. 39(10-11), 3075-3093 (2015). https://doi.org/10.1016/j.apm.2014.11.024

45. H. Jahanbakhti, A novel fractional-order neural network for model reduction of large-scale systems with fractional-order nonlinear structure. Soft. Comput. 24(17), 13 489-13 499 (2020). https://doi.org/10.1007/ s00500-020-04763-5

46. M.A.Z. Raja, J.A. Khan, I.M. Qureshi, A new stochastic approach for solution of Riccati differential equation of fractional order. Ann. Math. Artif. Intell. 60(3), 229-250 (2010)

47. S. Ghasemi, A. Nazemi, A fractional power series neural network for solving a class of fractional optimal control problems with equality and inequality constraints. Netw. Comput. Neural Syst. 30(1-4), 148-175 (2019). https://doi.org/10.1080/0954898X.2019.1693647

48. M. Yavari, A. Nazemi, An efficient numerical scheme for solving fractional infinite-horizon optimal control problems. ISA Trans. 94, 108-118 (2019). https://doi. org/10.1016/j.isatra.2019.04.016
49. Yavari, Nazemi, Fractional infinite-horizon optimal control problems with a feed forward neural network scheme. Netw. Comput. Neural Syst. 30(1-4), 125147 (2019). https://doi.org/10.1080/0954898X.2019. 1688878

50. H. Antil, R. Khatri, R.L. Lohner, D. Verma, Fractional deep neural network via constrained optimization. Mach. Learn. Sci. Technol. 2, 1-21 (2020)

51. N. Sadati, A. Ghaffarkhah, S. Ostadabbas, A new neural network based FOPID controller, in Proceedings of 2008 IEEE International Conference on Networking, Sensing and Control, ICNSC (2008), pp. 762-767

52. M. Zhou, Y. Feng, C. Xue, F. Han, Deep convolutional neural network based fractional-order terminal slidingmode control for robotic manipulators. Neurocomputing (2019). https://doi.org/10.1016/j.neucom.2019.04. 087 (online)

53. L. Chen, J. Qu, Y. Chai, R. Wu, G. Qi, Synchronization of a class of fractional-order chaotic neural networks. Entropy 15(8), 3265-3276 (2013)

54. M. Gai, S. Cui, S. Liang, X. Liu, Frequency distributed model of Caputo derivatives and robust stability of a class of multi-variable fractional-order neural networks with uncertainties. Neurocomputing 202, 91-97 (2016). https://doi.org/10.1016/j.neucom.2016.03.043

55. H. Liu, S.-G. Li, H.-X. Wang, G.-J. Li, Adaptive fuzzy synchronization for a class of fractionalorder neural networks. Chin. Phys. B 26(3), 030504 (2017). [Online]. http://www.stacks.iop. org $/ 1674-1056 / 26 / \mathrm{i}=3 / \mathrm{a}=030504$ ?key=crossref. de3f8b49032fccf86e10604042b47319

56. G. Li, H. Liu, Stability analysis and synchronization for a class of fractional-order neural networks. Entropy 18(2), 1-13 (2016)

57. X. Yang, C. Li, Q. Song, T. Huang, X. Chen, MittagLeffler stability analysis on variable-time impulsive fractional-order neural networks. Neurocomputing 207, 276-286 (2016). https://doi.org/10.1016/j. neucom.2016.04.045

58. H. Liu, Y. Pan, S. Li, Y. Chen, Synchronization for fractional-order neural networks with full/underactuation using fractional-order sliding mode control. Int. J. Mach. Learn. Cybern. 9(7), 1219-1232 (2018)

59. A. Wu, Z. Zeng, X. Song, Global Mittag-Leffler stabilization of fractional-order bidirectional associative memory neural networks. Neurocomputing 177, 489496 (2016)

60. S. Yang, C. Hu, J. Yu, H. Jiang, Exponential stability of fractional-order impulsive control systems with applications in synchronization. IEEE Trans. Cybern. 50(7), 3157-3168 (2020)

61. S. Yang, J. Yu, C. Hu, H. Jiang, Quasi-projective synchronization of fractional-order complex-valued recurrent neural networks. Neural Netw. 104, 104-113 (2018). https://doi.org/10.1016/j.neunet.2018.04.007

62. A. Wu, Z. Zeng, Boundedness, Mittag-Leffler stability and asymptotical $\omega$-periodicity of fractional-order fuzzy neural networks. Neural Netw. 74, 73-84 (2016). https://doi.org/10.1016/j.neunet.2015.11.003

63. S. Yang, J. Yu, C. Hu, H. Jiang, Finite-time synchronization of memristive neural networks with fractionalorder. IEEE Trans. Syst. Man Cybern. Syst. PP, 1-12 (2019) 
64. D. Lin, X. Chen, B. Li, X. Yang, LMI conditions for some dynamical behaviors of fractionalorder quaternion-valued neural networks. Adv. Differ. Equ. 1, 2019 (2019). https://doi.org/10.1186/ s13662-019-2207-0

65. A. Pratap, R. Raja, J. Alzabut, J. Dianavinnarasi, J. Cao, G. Rajchakit, Finite-time Mittag-Leffler stability of fractional-order quaternion-valued memristive neural networks with impulses. Neural Process. Lett. 51(2), 1485-1526 (2020). https://doi.org/10. 1007/s11063-019-10154-1

66. P. Liu, Z. Zeng, J. Wang, Multiple Mittag-Leffler stability of fractional-order recurrent neural networks. IEEE Trans. Syst. Man Cybern. Syst. 47(8), 2279-2288 (2017)

67. W. Li, Design and implement of neural network based fractional-order controller. in Robotic Welding, Intelligence and Automation (Springer, Berlin, Heidelberg, 2007), pp. 471-479

68. G.A.R. Ibraheem, A.T. Azar, I.K. Ibraheem, A.J. Humaidi, A novel design of a neural network-based fractional PID controller for mobile robots using hybridized fruit fly and particle swarm optimization. Complexity 1, 1-14 (2020)

69. F. Kheyrinataj, A. Nazemi, Fractional power series neural network for solving delay fractional optimal control problems. Connect. Sci. 32(1), 53-80 (2020). https://doi.org/10.1080/09540091.2019.1605498

70. M. Ahmad, E. Al-Solami, Evolving dynamic s-boxes using fractional-order Hopfield neural network based scheme. Entropy 22(7), 717 (2020)

71. E. Kaslik, S. Sivasundaram, Non-existence of periodic solutions in fractional-order dynamical systems and a remarkable difference between integer and fractionalorder derivatives of periodic functions. Nonlinear Anal. Real World Appl. 13(3), 1489-1497 (2012). https:// doi.org/10.1016/j.nonrwa.2011.11.013

72. I. Petráš, A note on the fractional-order cellular neural networks, in IEEE International Conference on Neural Networks-Conference Proceedings (IEEE), pp. 10211024 (2006)

73. V. Çelik, Bifurcation analysis of fractional order single cell with delay. Int. J. Bifurc. Chaos 25(2), 1-11 (2015)

74. C. Huang, J. Cao, Z. Ma, Delay-induced bifurcation in a tri-neuron fractional neural network. Int. J. Syst. Sci. 47(15), 3668-3677 (2016)

75. X. Yang, Q. Song, Y. Liu, Z. Zhao, Finite-time stability analysis of fractional-order neural networks with delay. Neurocomputing 152, 19-26 (2015). https://doi.org/ 10.1016/j.neucom.2014.11.023

76. S.M. Abedi Pahnehkolaei, A. Alfi, J.A. Machado, Uniform stability of fractional order leaky integrator echo state neural network with multiple time delays. Inf. Sci. 418-419, 703-716 (2017). https://doi.org/10.1016/j. ins.2017.08.046

77. C. Huang, J. Cao, Impact of leakage delay on bifurcation in high-order fractional BAM neural networks. Neural Netw. 98, 223-235 (2018). https://doi.org/10. 1016/j.neunet.2017.11.020

78. R. Ye, X. Liu, H. Zhang, J. Cao, Global MittagLeffler synchronization for fractional-order BAM neural networks with impulses and multiple variable delays via delayed-feedback control strategy. Neu- ral Process. Lett. (2019). https://doi.org/10.1007/ s11063-018-9801-0

79. R. Rakkiyappan, J. Cao, G. Velmurugan, Existence and uniform stability analysis of fractional-order complex-valued neural networks with time delays. IEEE Trans. Neural Netw. Learn. Syst. 26(1), 84-97 (2015)

80. R. Rakkiyappan, K. Udhayakumar, G. Velmurugan, J. Cao, A. Alsaedi, Stability and Hopf bifurcation analysis of fractional-order complex-valued neural networks with time delays. Adv. Differ. Equ. 1, 2017 (2017). https://doi.org/10.1186/s13662-017-1266-3

81. J. Yuan, L. Zhao, C. Huang, M. Xiao, Novel results on bifurcation for a fractional-order complex-valued neural network with leakage delay. Phys. A 514, 868-883 (2019). https://doi.org/10.1016/j.physa.2018.09.138

82. P. Mani, R. Rajan, L. Shanmugam, Y. Hoon Joo, Adaptive control for fractional order induced chaotic fuzzy cellular neural networks and its application to image encryption. Inf. Sci. 491, 74-89 (2019)

83. X. Huang, Z. Wang, Y. Li, Nonlinear dynamics and chaos in fractional-order Hopfield neural networks with delay. Adv. Math. Phys. 22, 1-16 (2013)

84. H. Wang, Y. Yu, G. Wen, Stability analysis of fractional-order Hopfield neural networks with time delays. Neural Netw. 55, 98-109 (2014). https://doi. org/10.1016/j.neunet.2014.03.012

85. R. Wu, Y. Lu, L. Chen, Finite-time stability of fractional delayed neural networks. Neurocomputing 149(PB), 700-707 (2015). https://doi.org/10.1016/j. neucom.2014.07.060

86. H. Wang, Y. Yu, G. Wen, S. Zhang, J. Yu, Global stability analysis of fractional-order Hopfield neural networks with time delay. Neurocomputing 154, 15-23 (2015). https://doi.org/10.1016/j.neucom.2014.12.031

87. H. Wang, Y. Yu, G. Wen, S. Zhang, Stability analysis of fractional-order neural networks with time delay. Neural Process. Lett. 42(2), 479-500 (2015)

88. H.P. Hu, J.K. Wang, F.L. Xie, Dynamics analysis of a new fractional-order Hopfield neural network with delay and its generalized projective synchronization. Entropy 21(1), 1-12 (2019)

89. F. Du, J.-G. Lu, New criteria on finite-time stability of fractional-order Hopfield neural networks with time delays. IEEE Trans. Neural Netw. Learn. Syst. 1, 1-9 (2020)

90. G. Velmurugan, R. Rakkiyappan, Hybrid projective synchronization of fractional-order neural networks with time delays, in Springer Proceedings in Mathematics and Statistics, vol. 143(1) (Springer Netherlands, 2015), pp. 645-655

91. X. Huang, Y. Fan, J. Jia, Z. Wang, Y. Li, Quasisynchronisation of fractional-order memristor-based neural networks with parameter mismatches. IET Control Theory Appl. 11(14), 2317-2327 (2017)

92. X. Yang, C. Li, T. Huang, Q. Song, X. Chen, Quasiuniform synchronization of fractional-order memristorbased neural networks with delay. Neurocomputing 234, 205-215 (2017). https://doi.org/10.1016/j. neucom.2017.01.014

93. J. Xiao, S. Zhong, Synchronization and stability of delayed fractional-order memristive quaternion-valued 
neural networks with parameter uncertainties. Neurocomputing 363, 321-338 (2019)

94. S.M.A. Pahnehkolaei, A. Alfi, J.A. Machado, Delay independent robust stability analysis of delayed fractional quaternion-valued leaky integrator echo state neural networks with QUAD condition. Appl. Math. Comput. 359, 278-293 (2019). https://doi.org/10. 1016/j.amc.2019.04.083

95. H. Zhang, R. Ye, J. Cao, A. Ahmed, X. Li, Y. Wan, Lyapunov functional approach to stability analysis of Riemann-Liouville fractional neural networks with time-varying delays. Asian J. Control 20(5), 1938-1951 (2018)

96. H. Zhang, M. Ye, J. Cao, A. Alsaedi, Synchronization Control of Riemann-Liouville fractional competitive network systems with time-varying delay and different time scales. Int. J. Control Autom. Syst. 16(3), 1404-1414 (2018)

97. C.J. Zúñiga-Aguilar, A. Coronel-Escamilla, J.F. Gómez-Aguilar, V.M. Alvarado-Martínez, H.M. Romero-Ugalde, New numerical approximation for solving fractional delay differential equations of variable order using artificial neural networks. Eur. Phys. J. Plus 133(2), 1-6 (2018)

98. F. Kheyrinataj, A. Nazemi, Fractional Chebyshev functional link neural network-optimization method for solving delay fractional optimal control problems with Atangana-Baleanu derivative. Optim. Control Appl. Methods 41(3), 808-832 (2020)

99. N.E. Tatar, Fractional Halanay inequality of order between one and two and application to neural network systems. Adv. Differ. Equ. 1, 2019 (2019). https://doi. org/10.1186/s13662-019-2208-Z

100. Z. Ding, Y. Shen, Global dissipativity of fractionalorder neural networks with time delays and discontinuous activations. Neurocomputing 196, 159-166 (2016). https://doi.org/10.1016/j.neucom.2016.03.005

101. W. Zhang, J. Cao, R. Wu, A. Alsaedi, F.E. Alsaadi, Projective synchronization of fractional-order delayed neural networks based on the comparison principle. Adv. Differ. Equ. 2018(1), 1-16 (2018). https://doi. org/10.1186/s13662-018-1530-1

102. G.C. Wu, T. Abdeljawad, J. Liu, D. Baleanu, K.T. Wu, Mittag-Leffler stability analysis of fractional discretetime neural networks via fixed point technique. Nonlinear Anal. Model. Control 24(6), 919-936 (2019)

103. Y. Cao, C. Bai, Finite-time stability of fractional-order bam neural networks with distributed delay. Abstr. Appl. Anal. 2014, 1-16 (2014)

104. A. Pratap, R. Raja, G. Rajchakit, J. Cao, O. Bagdasar, Mittag-Leffler state estimator design and synchronization analysis for fractional-order BAM neural networks with time delays. Int. J. Adapt. Control Signal Process. 33(5), 855-874 (2019)

105. Z. Yang, J. Zhang, Stability analysis of fractional-order bidirectional associative memory neural networks with mixed time-varying delays. Complexity 1, 1-17 (2019)

106. H. Qu, T. Zhang, J. Zhou, Global stability analysis of S-asymptotically $\omega$-periodic oscillation in fractionalorder cellular neural networks with time variable delays. Neurocomputing 399, 390-398 (2020). https:// doi.org/10.1016/j.neucom.2020.03.005
107. A. Pratap, R. Raja, J. Cao, C.P. Lim, O. Bagdasar, Stability and pinning synchronization analysis of fractional order delayed Cohen-Grossberg neural networks with discontinuous activations. Appl. Math. Comput. 359, 241-260 (2019). https://doi.org/10.1016/j.amc. 2019.04.062

108. C. Huang, J. Cao, M. Xiao, A. Alsaedi, T. Hayat, Bifurcations in a delayed fractional complex-valued neural network. Appl. Math. Comput. 292, 210-227 (2017). https://doi.org/10.1016/j.amc.2016.07.029

109. H.L. Li, C. Hu, J. Cao, H. Jiang, A. Alsaedi, Quasiprojective and complete synchronization of fractionalorder complex-valued neural networks with time delays. Neural Netw. 118, 102-109 (2019). https://doi. org/10.1016/j.neunet.2019.06.008

110. X. Wang, Z. Wang, X. Zhu, B. Meng, J. Xia, Stability and Hopf bifurcation of fractional-order complexvalued neural networks with time-delay. IEEE Access 7, 158 798-158 807 (2019)

111. X. You, Q. Song, Z. Zhao, Global Mittag-Leffler stability and synchronization of discrete-time fractionalorder complex-valued neural networks with time delay. Neural Netw. 122, 382-394 (2020). https://doi.org/10. 1016/j.neunet.2019.11.004

112. T. Hu, Z. He, X. Zhang, S. Zhong, Finite-time stability for fractional-order complex-valued neural networks with time delay. Appl. Math. Comput. 365, 124715 (2020). https://doi.org/10.1016/j.amc.2019.124715

113. R. Rakkiyappan, G. Velmurugan, X. Li, Complete stability analysis of complex-valued neural networks with time delays and impulses. Neural Process. Lett. 41(3), 435-468 (2015). https://doi.org/10.1016/j.chaos.2015. 08.003

114. A. Pratap, R. Raja, J. Cao, G. Rajchakit, H.M. Fardoun, Stability and synchronization criteria for fractional order competitive neural networks with time delays: an asymptotic expansion of Mittag Leffler function. J. Frankl. Inst. 356(4), 2212-2239 (2019). https://doi.org/10.1016/j.jfranklin.2019.01.017

115. A. Pratap, R. Raja, R.P. Agarwal, J. Cao, O. Bagdasar, Multi-weighted complex structure on fractional order coupled neural networks with linear coupling delay: a robust synchronization problem. Neural Process. Lett. 51(3), 2453-2479 (2020). https://doi.org/ 10.1007/s11063-019-10188-5

116. G. Velmurugan, R. Rakkiyappan, J. Cao, Finite-time synchronization of fractional-order memristor-based neural networks with time delays. Neural Netw. 73, 36-46 (2016). https://doi.org/10.1016/j.neunet.2015. 09.012

117. L. Chen, J. Cao, R. Wu, J.A. Tenreiro Machado, A.M. Lopes, H. Yang, Stability and synchronization of fractional-order memristive neural networks with multiple delays. Neural Netw. 94, 76-85 (2017). https:// doi.org/10.1016/j.neunet.2017.06.012

118. L. Zhang, Y. Yang, F. Wang, X. Sui, Lag synchronization for fractional-order memristive neural networks with time delay via switching jumps mismatch. J. Frankl. Inst. 355(3), 1217-1240 (2018). https://doi. org/10.1016/j.jfranklin.2017.12.017

119. J. Xiao, S. Zhong, Y. Li, F. Xu, Finite-time MittagLeffler synchronization of fractional-order memristive BAM neural networks with time delays. Neurocom- 
puting 219(2016), 431-439 (2017). https://doi.org/10. 1016/j.neucom.2016.09.049

120. M. Hui, C. Wei, J. Zhang, H.H.C. Iu, N. Luo, R. Yao, L. Bai, Finite-time synchronization of memristorbased fractional order Cohen-Grossberg neural networks. IEEE Access 8, 73 698-73 713 (2020)

121. C. Huang, X. Nie, X. Zhao, Q. Song, Z. Tu, M. Xiao, J. Cao, Novel bifurcation results for a delayed fractionalorder quaternion-valued neural network. Neural Netw. 117, 67-93 (2019). https://doi.org/10.1016/j.neunet. 2019.05.002

122. S. Thanompolkrang, W. Sawangtong, P. Sawangtong, Application of the generalized Laplace homotopy perturbation method to the time-fractional black-scholes equations based on the Katugampola fractional derivative in Caputo type. Computation 9(3), 33 (2021)

123. A. Alomari, G.A. Drabseh, M.F. Al-Jamal, R.B. AlBadarneh, Numerical simulation for fractional phi4 equation using homotopy Sumudu approach. Int. J. Simul. Process Model. 16(1), 26-33 (2021)

124. M. Johansyah, A. Supriatna, E. Rusyaman, J. Saputra, Bernoulli fractional differential equation solution using adomian decomposition method, in IOP Conference Series: Materials Science and Engineering, vol. 1115, no. 1 (IOP Publishing, 2021), p. 012015

125. M.Z. Mohamed, T.M. Elzaki, M.S. Algolam, E.M. Abd Elmohmoud, A.E. Hamza, New modified variational iteration Laplace transform method compares laplace adomian decomposition method for solution time-partial fractional differential equations. J. Appl. Math. 2021, 1-18 (2021)

126. M. Nadeem, J.-H. He, A. Islam, The homotopy perturbation method for fractional differential equations: part 1 Mohand transform. Int. J. Numer. Methods Heat Fluid Flow 1, 1-17 (2021)

127. A. Atangana, R.T. Alqahtani, New numerical method and application to Keller-Segel model with fractional order derivative. Chaos Solitons Fract. 116, 14-21 (2018)

128. R. Garrappa, Numerical solution of fractional differential equations: a survey and a software tutorial. Mathematics 6(2), 16 (2018)

129. M. Toufik, A. Atangana, New numerical approximation of fractional derivative with non-local and non-singular kernel: application to chaotic models. Eur. Phys. J. Plus 132(10), 1-16 (2017)

130. A. Atangana, S.İ Araz, New numerical method for ordinary differential equations: Newton polynomial. J. Comput. Appl. Math. 372, 112622 (2020)

131. S. Khan, J. Ahmad, I. Naseem, M. Moinuddin, A novel fractional gradient-based learning algorithm for recurrent neural networks. Circuits Syst. Signal Process. 37(2), 593-612 (2018)

132. J. Wang, Y. Wen, Y. Gou, Z. Ye, H. Chen, Fractionalorder gradient descent learning of BP neural networks with Caputo derivative. Neural Netw. 89, 19-30 (2017). https://doi.org/10.1016/j.neunet.2017.02.007

133. Y. Chen, G. Zhao, A Caputo-type fractional-order gradient descent learning of deep BP neural networks, in Proceedings of 2019 IEEE 3rd Advanced Information Management, Communicates, Electronic and Automation Control Conference, IMCEC 2019, no. Imcec (2019), pp. 546-550
134. D. Sheng, Y. Wei, Y. Chen, Y. Wang, Convolutional neural networks with fractional order gradient method. Neurocomputing 408, 42-50 (2019)

135. M.R. Chen, B.P. Chen, G.Q. Zeng, K.D. Lu, P. Chu, An adaptive fractional-order BP neural network based on extremal optimization for handwritten digits recognition. Neurocomputing 391, 260-272 (2020)

136. C.J. Zuñiga Aguilar, J.F. Gómez-Aguilar, V.M. Alvarado-Martínez, H.M. Romero-Ugalde, Fractional order neural networks for system identification. Chaos Solitons Fract. 130, 109444 (2020)

137. Y.F. Pu, Z. Yi, J.L. Zhou, Fractional Hopfield neural networks: fractional dynamic associative recurrent neural networks. IEEE Trans. Neural Netw. Learn. Syst. 28(10), 2319-2333 (2017)

138. Y.-F. PU, J. Wang, Fractional-order backpropagation neural networks: modified fractional-order steepest descent method for family of backpropagation neural networks, 1-18 (2019). arXiv:1906.09524

139. A. Sharafian, R. Ghasemi, Fractional neural observer design for a class of nonlinear fractional chaotic systems. Neural Comput. Appl. 31(4), 1201-1213 (2019)

140. H. Qu, X. Liu, A numerical method for solving fractional differential equations by using neural network. Adv. Math. Phys. 3, 2015 (2015)

141. A. Jafarian, M. Mokhtarpour, D. Baleanu, Artificial neural network approach for a class of fractional ordinary differential equation. Neural Comput. Appl. 28(4), 765-773 (2017)

142. S.S. Chaharborj, S.S. Chaharborj, Y. Mahmoudi, Study of fractional order integro-differential equations by using Chebyshev neural network. J. Math. Stat. 13(1), 1-13 (2017)

143. M.I. Nouh, Y.A. Azzam, E.A. Abdel-Salam, Modeling fractional polytropic gas spheres using artificial neural network. Neural Comput. Appl. (2020). https://doi. org/10.1007/s00521-020-05277-9

144. M.Ö. Efe, Neural network assisted computationally simple $\mathrm{PI}^{\lambda} \mathrm{D}^{\mu}$ control of a quadrotor UAV. IEEE Trans. Industr. Inf. 7(2), 354-361 (2011)

145. S. Wang, M. Yang, Y. Zhang, J. Li, L. Zou, S. Lu, B. Liu, J. Yang, Y. Zhang, Detection of left-sided and right-sided hearing loss via fractional Fourier transform. Entropy 18(5), 1-10 (2016)

146. S. Lodhi, M.A. Manzar, M.A.Z. Raja, Fractional neural network models for nonlinear Riccati systems. Neural Comput. Appl. 31, 359-378 (2019)

147. M.A.Z. Raja, J.A. Khan, I.M. Qureshi, Solution of fractional order system of Bagley-Torvik equation using evolutionary computational intelligence. Math. Probl. Eng. 2011, 1-15 (2011)

148. Javad Sabouri K, S. Effati, M. Pakdaman, A neural network approach for solving a class of fractional optimal control problems. Neural Process. Lett. 45(1), 5974 (2017)

149. A. Asgharnia, A. Jamali, R. Shahnazi, A. Maheri, Load mitigation of a class of 5-MW wind turbine with RBF neural network based fractional-order PID controller. ISA Trans. 96, 272-286 (2020). https://doi.org/10. 1016/j.isatra.2019.07.006

150. A. Shaikh, M. AsifJamal, F. Hanif, M. Sadiq AliKhan, S. Inayatullah, Neural minimization methods (NMM) for solving variable order fractional delay differential 
equations (FDDEs) with simulated annealing (SA). PLoS One 14(10), 1-22 (2019)

151. L. Zhang, Y. Yang, Optimal quasi-synchronization of fractional-order memristive neural networks with PSOA. Neural Comput. Appl. 32(13), 9667-9682 (2020). https://doi.org/10.1007/s00521-019-04488-z

152. A. Mohammadzadeh, E. Kayacan, A novel fractionalorder type-2 fuzzy control method for online frequency regulation in ac microgrid. Eng. Appl. Artif. Intell. 90(2018), 103483 (2020). https://doi.org/10.1016/j. engappai.2020.103483

153. Q. Chang, A. Hu, Y. Yang, L. Li, The optimization of synchronization control parameters for fractional-order delayed memristive neural networks using SIWPSO. Neural Process. Lett. 51(2), 1541-1556 (2020)

154. L. Zhang, Y. Yang, F. Wang, Synchronization analysis of fractional-order neural networks with time-varying delays via discontinuous neuron activations. Neurocomputing 275, 40-49 (2018)

155. H. Liu, S. Li, H. Wang, Y. Huo, J. Luo, Adaptive synchronization for a class of uncertain fractional-order neural networks. Entropy 17(10), 7185-7200 (2015)

156. S. Luo, S. Li, F. Tajaddodianfar, J. Hu, Adaptive synchronization of the fractional-order chaotic arch microelectro-mechanical system via Chebyshev neural network. IEEE Sens. J. 18(9), 3524-3532 (2018)

157. S. Song, X. Song, I.T. Balsera, Adaptive projective synchronization for fractional-order T-S fuzzy neural networks with time-delay and uncertain parameters. Optik 129, 140-152 (2017). https://doi.org/10.1016/j. ijleo.2016.10.067

158. X. Song, S. Song, I.T. Balsera, Mixed H $\mathrm{H} /$ passive projective synchronization for fractional-order neural networks with uncertain parameters and delays. Int. J. Innov. Comput. Inf. Control 13(4), 1273-1288 (2017)

159. H.B. Bao, J.D. Cao, Projective synchronization of fractional-order memristor-based neural networks. Neural Netw. 63, 1-9 (2015). https://doi.org/10.1016/ j.neunet.2014.10.007

160. J. Yu, C. Hu, H. Jiang, Corrigendum to "Projective synchronization for fractional neural networks ' '. Neural Netw. 67, 152-154 (2015). https://doi.org/10.1016/ j.neunet.2015.02.007

161. T.C. Lin, C.H. Kuo, H $\infty$ synchronization of uncertain fractional order chaotic systems: adaptive fuzzy approach. ISA Trans. 50(4), 548-556 (2011). https:// doi.org/10.1016/j.isatra.2011.06.001

162. J. Chen, C. Li, X. Yang, Global Mittag-Leffler projective synchronization of nonidentical fractional-order neural networks with delay via sliding mode control. Neurocomputing 313, 324-332 (2018). https:// doi.org/10.1016/j.neucom.2018.06.029

163. H. Wu, L. Wang, P. Niu, Y. Wang, Global projective synchronization in finite time of nonidentical fractional-order neural networks based on sliding mode control strategy. Neurocomputing 235(January), 264273 (2017). https://doi.org/10.1016/j.neucom.2017.01. 022

164. Y. Xi, Y. Yu, S. Zhang, X. Hai, Finite-time robust control of uncertain fractional-order Hopfield neural networks via sliding mode control. Chin. Phys. B 27(1), 010202 (2018)
165. N. Liu, J. Fei, Adaptive fractional sliding mode control of active power filter based on dual RBF neural networks. IEEE Access 5, 27 590-27 598 (2017)

166. B. Meng, X. Wang, Adaptive synchronization for uncertain delayed fractional-order hopfield neural networks via fractional-order sliding mode control. Math. Probl. Eng. 2014, 1-17 (2018)

167. H. Bao, J.H. Park, J. Cao, Synchronization of fractional-order complex-valued neural networks with time delay. Neural Netw. 81, 16-28 (2016). https:// doi.org/10.1016/j.neunet.2016.05.003

168. J. Zhang, J. Wu, H. Bao, J. Cao, Synchronization analysis of fractional-order three-neuron BAM neural networks with multiple time delays. Appl. Math. Comput. 339, 441-450 (2018). https://doi.org/10.1016/j. amc.2018.06.013

169. T. Ma, J. Zhang, Hybrid synchronization of coupled fractional-order complex networks. Neurocomputing 157, 166-172 (2015). https://doi.org/10.1016/j. neucom.2015.01.022

170. S. Zhou, H. Li, Z. Zhu, Chaos control and synchronization in a fractional neuron network system. Chaos Solitons Fract. 36(4), 973-984 (2008)

171. J. Yu, C. Hu, H. Jiang, $\alpha$-stability and $\alpha$ synchronization for fractional-order neural networks. Neural Netw. 35, 82-87 (2012). https://doi.org/10. 1016/j.neunet.2012.07.009

172. L. Zhang, Y. Yang, F. Wang, Lag synchronization for fractional-order memristive neural networks via period intermittent control. Nonlinear Dyn. 89(1), 367-381 (2017)

173. W. Zhang, R. Wu, J. Cao, A. Alsaedi, T. Hayat, Synchronization of a class of fractional-order neural networks with multiple time delays by comparison principles. Nonlinear Anal. Model. Control 22(5), 636-645 (2017)

174. L. Chen, R. Wu, J. Cao, J.B. Liu, Stability and synchronization of memristor-based fractional-order delayed neural networks. Neural Netw. 71, 37-44 (2015). https://doi.org/10.1016/j.neunet.2015.07.012

175. M. Zheng, L. Li, H. Peng, J. Xiao, Y. Yang, H. Zhao, Finite-time stability and synchronization for memristor-based fractional-order Cohen-Grossberg neural network. Eur. Physi. J. B 89(9), 1 (2016)

176. M. Zheng, L. Li, H. Peng, J. Xiao, Y. Yang, Y. Zhang, H. Zhao, Finite-time stability and synchronization of memristor-based fractional-order fuzzy cellular neural networks. Commun. Nonlinear Sci. Numer. Simul. 59, 272-291 (2018). https://doi.org/10.1016/j.cnsns.2017. 11.025

177. M. Zheng, L. Li, H. Peng, J. Xiao, Y. Yang, H. Zhao, Finite-time projective synchronization of memristorbased delay fractional-order neural networks. Nonlinear Dyn. 89(4), 2641-2655 (2017)

178. L. Zhang, Y. Yang, F. Wang, Projective synchronization of fractional-order memristive neural networks with switching jumps mismatch. Phys. A 471, 402-415 (2017). https://doi.org/10.1016/j.physa.2016.12.030

179. F. Zouari, A. Boulkroune, A. Ibeas, Neural adaptive quantized output-feedback control-based synchronization of uncertain time-delay incommensurate fractional-order chaotic systems with input nonlinear- 
ities. Neurocomputing 237, 200-225 (2017). https:// doi.org/10.1016/j.neucom.2016.11.036

180. Y. Gu, Y. Yu, H. Wang, Synchronization for fractionalorder time-delayed memristor-based neural networks with parameter uncertainty. J. Frankl. Inst. 353(15), 3657-3684 (2016). https://doi.org/10.1016/j.jfranklin. 2016.06.029

181. Z. Ding, Y. Shen, L. Wang, Global Mittag-Leffler synchronization of fractional-order neural networks with discontinuous activations. Neural Netw. 73, 77-85 (2016). https://doi.org/10.1016/j.neunet.2015.10.010

182. X. Li, J.-A. Fang, W. Zhang, H. Li, Finite-time synchronization of fractional-order memristive recurrent neural networks with discontinuous activation functions R. Neurocomputing 316, 284-293 (2018). https://doi.org/10.1016/j.neucom.2018.08.003

183. W. Zhang, J. Cao, D. Chen, F.E. Alsaadi, Synchronization in fractional-order complex-valued delayed neural networks. Entropy 20(1), 1-16 (2018)

184. H. Bao, J.H. Park, J. Cao, Adaptive synchronization of fractional-order memristor-based neural networks with time delay. Nonlinear Dyn. 82(3), 1343-1354 (2015)

185. I. Stamova, Global Mittag-Leffler stability and synchronization of impulsive fractional-order neural networks with time-varying delays. Nonlinear Dyn. 77(4), 1251-1260 (2014)

186. I. Stamova, G. Stamov, Mittag-Leffler synchronization of fractional neural networks with time-varying delays and reaction-diffusion terms using impulsive and linear controllers. Neural Netw. 96, 22-32 (2017). https:// doi.org/10.1016/j.neunet.2017.08.009

187. S. Zhou, X. Lin, H. Li, Chaotic synchronization of a fractional-order system based on washout filter control. Commun. Nonlinear Sci. Numer. Simul. 16(3), 15331540 (2011). https://doi.org/10.1016/j.cnsns.2010.06. 022

188. X. Zhang, C. Yang, Neural network synchronization of fractional-order chaotic systems subject to backlash nonlinearity. AIP Adv. (2020). https://doi.org/10. 1063/5.0007914

189. G. Li, C. Sun, Adaptive neural network backstepping control of fractional-order Chua-Hartley chaotic system. Adv. Differ. Equ. 1, 2019 (2019). https://doi.org/ 10.1186/s13662-019-2099-z

190. Y. Kao, Y. Li, J.H. Park, X. Chen, Mittag-Leffler synchronization of delayed fractional memristor neural networks via adaptive control. IEEE Trans. Neural Netw. Learn. Syst. 1, 1-6 (2020)

191. X. Zhang, X. Zhang, D. Li, D. Yang, Adaptive synchronization for a class of fractional order time-delay uncertain chaotic systems via fuzzy fractional order neural network. Int. J. Control Autom. Syst. 17(106112016), 1-12 (2019)

192. W. Cheng, A. Wu, J.E. Zhang, B. Li, Adaptive control of Mittag-Leffler stabilization and synchronization for delayed fractional-order BAM neural networks. Adv. Differ. Equ. 1, 2019 (2019). https://doi.org/10.1186/ s13662-019-2279-x

193. J. He, F. Chen, T. Lei, Q. Bi, Global adaptive matrixprojective synchronization of delayed fractional-order competitive neural network with different time scales. Neural Comput. Appl. 32(16), 12 813-12 826 (2020). https://doi.org/10.1007/s00521-020-04728-7
194. B. Meng, Z. Wang, Z. Wang, Adaptive sliding mode control for a class of uncertain nonlinear fractionalorder Hopfield neural networks. AIP Adv. 10(1063/1), 5097374 (2019)

195. S. Song, X. Song, I. Tejado, Projective synchronization for two nonidentical time-delayed fractional-order $\mathrm{T}-\mathrm{S}$ fuzzy neural networks based on mixed $\mathrm{H} \infty$ /passive adaptive sliding mode control. Int. J. Mach. Learn. Cybern. 10(5), 799-812 (2019). https://doi.org/10. 1007/s13042-017-0761-x

196. Y.L. Wang, H. Jahanshahi, S. Bekiros, F. Bezzina, Y.M. Chu, A.A. Aly, Deep recurrent neural networks with finite-time terminal sliding mode control for a chaotic fractional-order financial system with market confidence. Chaos Solitons Fract. 146, 110881 (2021). https://doi.org/10.1016/j.chaos.2021.110881

197. A. Sharafian, A. Sharifi, W. Zhang, Fractional sliding mode based on RBF neural network observer: application to HIV infection mathematical model. Comput. Math. Appl. 79(11), 3179-3188 (2020). https://doi. org/10.1016/j.camwa.2020.01.014

198. J. Fei, H. Wang, Experimental investigation of recurrent neural network fractional-order sliding mode control of active power filter. IEEE Trans. Circuits Syst. II Expr. Briefs PP(c), 1-1 (2019)

199. Fei, Wang, Recurrent neural network fractional-order sliding mode control of dynamic systems. J. Frankl. Inst. 357(8), 4574-4591 (2020). https://doi.org/10. 1016/j.jfranklin.2020.01.050

200. Z. Han, S. Li, H. Liu, Composite learning sliding mode synchronization of chaotic fractional-order neural networks. J. Adv. Res. (2020). https://doi.org/10.1016/j. jare.2020.04.006

201. W. Wang, X. Jia, Z. Wang, X. Luo, L. Li, J. Kurths, M. Yuan, Fixed-time synchronization of fractional order memristive MAM neural networks by sliding mode control. Neurocomputing 401, 364-376 (2020)

202. A. Pratap, R. Raja, J. Cao, F.A. Rihan, A.R. Seadawy, Quasi-pinning synchronization and stabilization of fractional order BAM neural networks with delays and discontinuous neuron activations. Chaos Solitons Fract. 131, 109491 (2020)

203. M.V. Thuan, T.N. Binh, D.C. Huong, Finite-time guaranteed cost control of Caputo fractional-order neural networks. Asian J. Control 22(2), 696-705 (2020)

204. T. Hu, Z. He, X. Zhang, S. Zhong, Global synchronization of time-invariant uncertainty fractional-order neural networks with time delay. Neurocomputing $\mathbf{3 3 9}$, 45-58 (2019). https://doi.org/10.1016/j.neucom.2019. 02.020

205. P. Liu, M. Kong, Z. Zeng, Projective synchronization analysis of fractional-order neural networks with mixed time delays. IEEE Trans. Cybern. 1, 1-11 (2020)

206. H. Yan, Y. Qiao, L. Duan, L. Zhang, Global MittagLeffler stabilization of fractional-order BAM neural networks with linear state feedback controllers. Math. Probl. Eng. 2020, 1-14 (2020)

207. Z. Yang, J. Li, Y. Niu, Finite-time stabilization of fractional-order delayed bidirectional associative memory neural networks. ScienceAsia 45(6), 589-596 (2019)

208. Y. Fan, X. Huang, Z. Wang, J. Xia, H. Shen, Quantized control for synchronization of delayed fractionalorder memristive neural networks. Neural Process. 
Lett. 52(1), 403-419 (2020). https://doi.org/10.1007/ s11063-020-10259-y

209. Y. Gu, Y. Yu, H. Wang, Projective synchronization for fractional-order memristor-based neural networks with time delays. Neural Comput. Appl. 31(10), 6039-6054 (2019). https://doi.org/10.1007/s00521-018-3391-7

210. J. Jia, X. Huang, Y. Li, J. Cao, A. Alsaedi, Global stabilization of fractional-order memristor-based neural networks with time delay. IEEE Trans. Neural Netw. Learn. Syst. 31(3), 997-1009 (2020)

211. C. Chen, Z. Ding, S. Li, L. Wang, Synchronization of fractional-order memristive neural networks with time delays, in Proceedings - 2019 Chinese Automation Congress. CAC 2019 (2019), pp. 2754-2759

212. L. Chen, T. Huang, J.A. Tenreiro Machado, A.M. Lopes, Y. Chai, R. Wu, Delay-dependent criterion for asymptotic stability of a class of fractional-order memristive neural networks with time-varying delays. Neural Netw. 118, 289-299 (2019). https://doi.org/10. 1016/j.neunet.2019.07.006

213. J. Xiao, J. Cao, J. Cheng, S. Zhong, S. Wen, Novel methods to finite-time Mittag-Leffler synchronization problem of fractional-order quaternion-valued neural networks. Inf. Sci. 526, 221-244 (2020). https://doi. org/10.1016/j.ins.2020.03.101

214. J. Xiao, S. Wen, X. Yang, S. Zhong, New approach to global Mittag-Leffler synchronization problem of fractional-order quaternion-valued BAM neural networks based on a new inequality. Neural Netw. 122, 320-337 (2020). https://doi.org/10.1016/ j.neunet.2019.10.017

215. G. Rajchakit, P. Chanthorn, P. Kaewmesri, R. Sriraman, C.P. Lim, Global Mittag-Leffler stability and stabilization analysis of fractional-order quaternionvalued memristive neural networks. Mathematics 8(3), 422 (2020)

216. H.L. Li, L. Zhang, C. Hu, H. Jiang, J. Cao, Global Mittag-Leffler synchronization of fractionalorder delayed quaternion-valued neural networks: direct quaternion approach. Appl. Math. Comput. 373, $125020(2020)$

217. S.A. Karthick, R. Sakthivel, Y.K. Ma, S. Mohanapriya, A. Leelamani, Disturbance rejection of fractional-order T-S fuzzy neural networks based on quantized dynamic output feedback controller. Appl. Math. Comput. 361, 846-857 (2019)

218. A. Pratap, R. Raja, J. Cao, J. Alzabut, C. Huang, Finite-time synchronization criterion of graph theory perspective fractional-order coupled discontinuous neural networks. Adv. Differ. Equ. 1, 2020 (2020). https:// doi.org/10.1186/s13662-020-02551-x

219. C. Wang, Q. Yang, Y. Zhuo, R. Li, Synchronization analysis of a fractional-order non-autonomous neural network with time delay. Phys. A 549, 124176 (2020). https://doi.org/10.1016/j.physa.2020.124176

220. Y. Gu, H. Wang, Y. Yu, Stability and synchronization for Riemann-Liouville fractional-order time-delayed inertial neural networks. Neurocomputing 340, 270280 (2019). https://doi.org/10.1016/j.neucom.2019.03. 005

221. Y. Gu, H. Wang, Y. Yu, Synchronization for fractionalorder discrete-time neural networks with time delays. Appl. Math. Comput. 372, 124995 (2020)
222. M. Syed Ali, M. Hymavathi, S. Senan, V. Shekher, S. Arik, Global asymptotic synchronization of impulsive fractional-order complex-valued memristor-based neural networks with time varying delays. Commun. Nonlinear Sci. Numer. Simul. 78, 104869 (2019). https:// doi.org/10.1016/j.cnsns.2019.104869

223. G. Rajchakit, A. Pratap, R. Raja, J. Cao, J. Alzabut, C. Huang, Hybrid control scheme for projective lag synchronization of Riemann-Liouville sense fractional order memristive BAM neural networks with mixed delays. Mathematics 7(8), 759 (2019)

224. A. Pratap, R. Raja, R.P. Agarwal, J. Cao, Stability analysis and robust synchronization of fractional-order competitive neural networks with different time scales and impulsive perturbations. Int. J. Adapt. Control Signal Process. 33(11), 1635-1660 (2019)

225. Z. Yang, J. Zhang, Global stabilization of fractionalorder bidirectional associative memory neural networks with mixed time delays via adaptive feedback control. Int. J. Comput. Math. 0(0), 1-17 (2019). https://doi. org/10.1080/00207160.2019.1677897

226. P. Anbalagan, R. Ramachandran, J. Cao, G. Rajchakit, C.P. Lim, Global Robust synchronization of fractional order complex valued neural networks with mixed time varying delays and impulses. Int. J. Control Autom. Syst. 17(2), 509-520 (2019)

227. P. Wan, J. Jian, Impulsive stabilization and synchronization of fractional-order complex-valued neural networks. Neural Process. Lett. 50(3), 2201-2218 (2019). https://doi.org/10.1007/s11063-019-10002-2

228. L. Zhang, Y. Yang, Finite time impulsive synchronization of fractional order memristive BAM neural networks. Neurocomputing 384, 213-224 (2020). https:// doi.org/10.1016/j.neucom.2019.12.056

229. L. Zhang, Stability analysis of fractional order memristive discontinuous neural networks with partial state control. Phys. A 531(18), 121756 (2019). https://doi. org/10.1016/j.physa.2019.121756

230. J. Cao, G. Stamov, I. Stamova, S. Simeonov, Almost periodicity in impulsive fractional-order reactiondiffusion neural networks with time-varying delays. IEEE Trans. Cybern. 3, 1-11 (2020)

231. A. Pratap, R. Raja, J. Alzabut, J. Cao, G. Rajchakit, C. Huang, Mittag-Leffler stability and adaptive impulsive synchronization of fractional order neural networks in quaternion field. Math. Methods Appl. Sci. 43(10), 6223-6253 (2020)

232. Z.B. Wu, Y.Z. Zou, N.J. Huang, A system of fractionalorder interval projection neural networks. J. Comput. Appl. Math. 294, 389-402 (2016). https://doi.org/10. 1016/j.cam.2015.09.007

233. L. Zhang, Q. Song, Z. Zhao, Stability analysis of fractional-order complex-valued neural networks with both leakage and discrete delays. Appl. Math. Comput. 298, 296-309 (2017). https://doi.org/10.1016/j. amc.2016.11.027

234. S. Tyagi, S. Abbas, M. Hafayed, Global Mittag-Leffler stability of complex valued fractional-order neural network with discrete and distributed delays. Rendiconti del Circolo Matematico di Palermo 65(3), 485-505 (2016) 
235. Y. Cao, C. Bai, Existence and stability analysis of fractional order BAM neural networks with a time delay. Appl. Math. 06(12), 2057-2068 (2015)

236. X. Yang, Q. Song, Y. Liu, Z. Zhao, Uniform stability analysis of fractional-order bam neural networks with delays in the leakage terms. Abstr. Appl. Anal. 2014, $1-15(2014)$

237. Y. Yang, Y. He, Y. Wang, M. Wu, Stability analysis of fractional-order neural networks: an LMI approach. Neurocomputing 285, 82-93 (2018). https://doi.org/ 10.1016/j.neucom.2018.01.036

238. Y. Zhao, Y. Cai, G. Fan, Dynamical behavior for fractional-order shunting inhibitory cellular neural networks. J. Nonlinear Sci. Appl. 09(06), 4589-4599 (2018)

239. R. Rakkiyappan, G. Velmurugan, J. Cao, Stability analysis of memristor-based fractional-order neural networks with different memductance functions. Cogn. Neurodyn. 9(2), 145-177 (2015)

240. S. Zhang, Y. Yu, H. Wang, Mittag-Leffler stability of fractional-order Hopfield neural networks. Nonlinear Anal. Hybrid Syst 16(11371049), 104-121 (2015). https://doi.org/10.1016/j.nahs.2014.10.001

241. C. Song, J. Cao, Dynamics in fractional-order neural networks. Neurocomputing 142, 494-498 (2014). https://doi.org/10.1016/j.neucom.2014.03.047

242. L. Chen, Y. Chai, R. Wu, T. Ma, H. Zhai, Dynamic analysis of a class of fractional-order neural networks with delay. Neurocomputing 111, 190-194 (2013). https://doi.org/10.1016/j.neucom.2012.11.034

243. X. Ding, J. Cao, X. Zhao, F.E. Alsaadi, Finite-time stability of fractional-order complex-valued neural networks with time delays. Neural Process. Lett. 46(2), 561-580 (2017)

244. L. Wang, Q. Song, Y. Liu, Z. Zhao, F.E. Alsaadi, Finite-time stability analysis of fractional-order complex-valued memristor-based neural networks with both leakage and time-varying delays. Neurocomputing 245, 86-101 (2017). https://doi.org/10.1016/j. neucom.2017.03.042

245. Y. Ke, C. Miao, Stability analysis of fractional-order Cohen-Grossberg neural networks with time delay. Int. J. Comput. Math. 92(6), 1102-1113 (2015)

246. L. Chen, C. Liu, R. Wu, Y. He, Y. Chai, Finite-time stability criteria for a class of fractional-order neural networks with delay. Neural Comput. Appl. 27(3), 549-556 (2015)

247. A. Alofi, J. Cao, A. Elaiw, A. Al-Mazrooei, Delaydependent stability criterion of caputo fractional neural networks with distributed delay. Discrete Dyn. Nat. Soc. 1, 1-15 (2014)

248. Z. Ding, Z. Zeng, L. Wang, Robust finite-time stabilization of fractional-order neural networks with discontinuous and continuous activation functions under uncertainty. IEEE Trans. Neural Netw. Learn. Syst. 29(5), 1477-1490 (2018)

249. C. Huang, Y. Meng, J. Cao, A. Alsaedi, F.E. Alsaadi, New bifurcation results for fractional BAM neural network with leakage delay. Chaos Solitons Fract. 100, 31-44 (2017)

250. C. Huang, J. Cao, M. Xiao, A. Alsaedi, T. Hayat, Effects of time delays on stability and Hopf bifurcation in a fractional ring-structured network with arbi- trary neurons. Commun. Nonlinear Sci. Numer. Simul. 57, 1-13 (2018). https://doi.org/10.1016/j.cnsns.2017. 09.005

251. E. Kaslik, I.R. Rădulescu, Dynamics of complex-valued fractional-order neural networks. Neural Netw. 89, 39-49 (2017). https://doi.org/10.1016/j.neunet.2017. 02.011

252. H. Wu, X. Zhang, S. Xue, P. Niu, Quasi-uniform stability of Caputo-type fractional-order neural networks with mixed delay. Int. J. Mach. Learn. Cybern. 8(5), 1501-1511 (2017)

253. N. Sene, Fractional input stability and its application to neural network. Discrete Cont. Dyn. Syst. 13, 853865 (2018)

254. R. Rakkiyappan, R. Sivaranjani, G. Velmurugan, J. Cao, Analysis of global $\mathrm{O}(\mathrm{t}-\alpha)$ stability and global asymptotical periodicity for a class of fractional-order complex-valued neural networks with time varying delays. Neural Netw. 77, 51-69 (2016). https://doi. org/10.1016/j.neunet.2016.01.007

255. H. Zhang, R. Ye, J. Cao, A. Alsaedi, Existence and globally asymptotic stability of equilibrium solution for fractional-order hybrid BAM neural networks with distributed delays and impulses. Complexity 2017, 1-15 (2017)

256. H. Wu, X. Zhang, S. Xue, L. Wang, Y. Wang, LMI conditions to global Mittag-Leffler stability of fractionalorder neural networks with impulses. Neurocomputing 193, 148-154 (2016). https://doi.org/10.1016/j. neucom.2016.02.002

257. G. Velmurugan, R. Rakkiyappan, V. Vembarasan, J. Cao, A. Alsaedi, Dissipativity and stability analysis of fractional-order complex-valued neural networks with time delay. Neural Netw. 86, 42-53 (2017). https:// doi.org/10.1016/j.neunet.2016.10.010

258. G. Stamov, I. Stamova, Impulsive fractional-order neural networks with time-varying delays: almost periodic solutions. Neural Comput. Appl. 28(11), 3307-3316 (2017)

259. F. Wang, Y. Yang, M. Hu, Asymptotic stability of delayed fractional-order neural networks with impulsive effects. Neurocomputing 154, 239-244 (2015). https://doi.org/10.1016/j.neucom.2014.11.068

260. R.P. Agarwal, S. Hristova, D. O'regan, Lyapunov functions to Caputo reaction-diffusion fractional neural networks with time-varying delays. J. Math. Comput. Sci. 18(3), 328-345 (2018)

261. S. Zhang, Y. Yu, Q. Wang, Stability analysis of fractional-order Hopfield neural networks with discontinuous activation functions. Neurocomputing 171, 1075-1084 (2016). https://doi.org/10.1016/j.neucom. 2015.07.077

262. B. Chen, J. Chen, Global asymptotical $\omega$-periodicity of a fractional-order non-autonomous neural networks. Neural Netw. 68, 78-88 (2015). https://doi.org/10. 1016/j.neunet.2015.04.006

263. S. Mehdi, A. Pahnehkolaei, A. Alfi, J.A.T. Machado, Dynamic stability analysis of fractional order leaky integrator echo state neural networks. Commun. Nonlinear Sci. Numer. Simul. 47, 328-337 (2017). https:// doi.org/10.1016/j.cnsns.2016.11.013

264. H. Zhang, R. Ye, J. Cao, A. Alsaedi, Delay-independent stability of Riemann-Liouville fractional neutral-type 
delayed neural networks. Neural Process. Lett. 47(2), 427-442 (2017)

265. S. Liang, R. Wu, L. Chen, Comparison principles and stability of nonlinear fractional-order cellular neural networks with multiple time delays. Neurocomputing 168, 618-625 (2015). https://doi.org/10.1016/j. neucom.2015.05.063

266. H. Wei, R. Li, C. Chen, Z. Tu, Stability Analysis of fractional order complex-valued memristive neural networks with time delays. Neural Process. Lett. 45(2), 379-399 (2017)

267. A. Wu, L. Liu, T. Huang, Z. Zeng, Mittag-Leffler stability of fractional-order neural networks in the presence of generalized piecewise constant arguments. Neural Netw. 85, 118-127 (2017). https://doi.org/10.1016/ j.neunet.2016.10.002

268. R. Li, J. Cao, A. Alsaedi, F. Alsaadi, Stability analysis of fractional-order delayed neural networks. Nonlinear Anal. Model. Control 22(4), 505-520 (2017)

269. B. Chen, J. Chen, Global $\mathrm{O}(\mathrm{t} \alpha)$ stability and global asymptotical periodicity for a non-autonomous fractional-order neural networks with time-varying delays. Neural Netw. 73, 47-57 (2016). https://doi. org/10.1016/j.neunet.2015.09.007

270. H. Zhang, M. Ye, R. Ye, J. Cao, Synchronization stability of Riemann-Liouville fractional delay-coupled complex neural networks. Phys. A 508, 155-165 (2018). https://doi.org/10.1016/j.physa.2018.05.060

271. M. Tuz, G.A. Suroglu, Anti-periodic solutions for fractional-order bidirectional associative memory neural networks with delays. Therm. Sci. 23, S2169-S2177 (2019)

272. X. Yao, S. Zhong, T. Hu, H. Cheng, D. Zhang, Uniformly stable and attractive of fractional-order memristor-based neural networks with multiple delays. Appl. Math. Comput. 347, 392-403 (2019). https:// doi.org/10.1016/j.amc.2018.11.028

273. M. Syed Ali, G. Narayanan, V. Shekher, H. Alsulami, T. Saeed, Dynamic stability analysis of stochastic fractional-order memristor fuzzy BAM neural networks with delay and leakage terms. Appl. Math. Comput. 369, 124896 (2020). https://doi.org/10.1016/j. amc.2019.124896

274. J. Alzabut, S. Tyagi, S. Abbas, Discrete fractionalorder BAM neural networks with leakage delay: existence and stability results. Asian J. Control 22(1), 143155 (2020)

275. A. Jmal, A. Ben Makhlouf, A.M. Nagy, O. Naifar, Finite-time stability for Caputo-Katugampola fractional-order time-delayed neural networks. Neural Process. Lett. 50(1), 607-621 (2019). https://doi.org/ 10.1007/s11063-019-10060-6

276. X. You, Q. Song, Z. Zhao, Existence and finite-time stability of discrete fractional-order complex-valued neural networks with time delays. Neural Netw. 123, 248260 (2020). https://doi.org/10.1016/j.neunet.2019.12. 012

277. M. Syed Ali, G. Narayanan, Z. Orman, V. Shekher, S. Arik, Finite time stability analysis of fractional-order complex-valued memristive neural networks with proportional delays. Neural Process. Lett. 51(1), 407-426 (2020). https://doi.org/10.1007/s11063-019-10097-7
278. G. Nagamani, M. Shafiya, G. Soundararajan, M. Prakash, Robust state estimation for fractional-order delayed BAM neural networks via LMI approach. J. Frankl. Inst. 357(8), 4964-4982 (2020)

279. W. Liu, M. Jiang, M. Yan, Stability analysis of memristor-based time-delay fractional-order neural networks. Neurocomputing 323, 117-127 (2019). https://doi.org/10.1016/j.neucom.2018.09.073

280. M. Syed Ali, G. Narayanan, S. Sevgen, V. Shekher, S. Arik, Global stability analysis of fractional-order fuzzy BAM neural networks with time delay and impulsive effects. Commun. Nonlinear Sci. Numer. Simul. 78, 104853 (2019). https://doi.org/10.1016/j.cnsns.2019. 104853

281. C. Huang, X. Zhao, X. Wang, Z. Wang, M. Xiao, J. Cao, Disparate delays-induced bifurcations in a fractional-order neural network. J. Frankl. Inst. 356(5), 2825-2846 (2019). https://doi.org/10.1016/j. jfranklin.2018.11.027

282. C. Xu, M. Liao, P. Li, J. Yan, Influence of time delay on bifurcation in fractional order BAM neural networks with four delays. IEEE Access 7, 70 955-70 965 (2019)

283. P. Wan, D. Sun, M. Zhao, H. Zhao, Monostability and multistability for almost-periodic solutions of fractional-order neural networks with unsaturating piecewise linear activation functions. IEEE Tran. Neural Netw. Learn. Syst. 1, 1-15 (2020)

284. L. Wan, X. Zhan, H. Gao, Q. Yang, T. Han, M. Ye, Multiple asymptotical stability analysis for fractionalorder neural networks with time delays. Int. J. Syst. Sci. 50(10), 2063-2076 (2019). https://doi.org/10. 1080/00207721.2019.1646836

285. G. Rajchakit, P. Chanthorn, M. Niezabitowski, R. Raja, D. Baleanu, A. Pratap, Impulsive effects on stability and passivity analysis of memristor-based fractional-order competitive neural networks. Neurocomputing 417, 290-301 (2020). https://doi.org/10. 1016/j.neucom.2020.07.036

286. G.A. Anastassiou, Fractional neural network approximation. Comput. Math. Appl. 64(6), 1655-1676 (2012). https://doi.org/10.1016/j.camwa.2012.01.019

287. R.W. Ibrahim, The fractional differential polynomial neural network for approximation of functions. Entropy 15(10), 4188-4198 (2013)

288. S. Lu, X. Wang, Observer-based command filtered adaptive neural network tracking control for fractionalorder chaotic PMSM. IEEE Access 7, 88 777-88 788 (2019)

289. S. Lu, X. Wang, Y. Li, Adaptive neural network control for fractional-order PMSM with time delay based on command filtered backstepping. AIP Adv. 10(1063/1), 5094574 (2019)

290. D. Baleanu, Z.B. Güvenç, J.T. Machado (eds.), New Trends in Nanotechnology and Fractional Calculus Applications (Springer, New York, 2010), p. C397

291. Y. Fan, X. Huang, Z. Wang, Y. Li, Nonlinear dynamics and chaos in a simplified memristor-based fractionalorder neural network with discontinuous memductance function. Nonlinear Dyn. 93(2), 611-627 (2018). https://doi.org/10.1007/s11071-018-4213-2

292. A.H. Tian, C.B. Fu, X.Y. Su, H.T. Yau, Lathe tool chatter vibration diagnostic using general regression neural network based on Chua's circuit and fractional- 
order Lorenz master/slave chaotic system. J. Low Freq. Noise Vib. Act. Control 38(3-4), 953-966 (2019)

293. L. Zhang, Y. Yang, Stability analysis of fractional order Hopfield neural networks with optimal discontinuous control. Neural Process. Lett. 50(1), 581-593 (2019). https://doi.org/10.1007/s11063-019-10054-4

294. L. Chen, H. Yin, T. Huang, L. Yuan, S. Zheng, L. Yin, Chaos in fractional-order discrete neural networks with application to image encryption. Neural Netw. 125, 174-184 (2020). https://doi.org/10.1016/ j.neunet.2020.02.008

295. X. Wang, Y. Su, C. Luo, C. Wang, A novel image encryption algorithm based on fractional order 5D cellular neural network and Fisher-Yates scrambling. PLoS ONE 15(7), 1-18 (2020). https://doi.org/10. 1371/journal.pone.0236015

296. A. Boroomand, M.B. Menhaj, ON-line nonlinear systems identification of coupled tanks via fractional differential neural networks, in 2009 Chinese Control and Decision Conference, pp. 2185-2189 (2009)

297. H. Bao, J. Cao, J. Kurths, State estimation of fractional-order delayed memristive neural networks. Nonlinear Dyn. 94(2), 1215-1225 (2018). https://doi. org/10.1007/s11071-018-4419-3

298. R. Li, X. Gao, J. Cao, Non-fragile state estimation for delayed fractional-order memristive neural networks. Appl. Math. Comput. 340, 221-233 (2019). https:// doi.org/10.1016/j.amc.2018.08.031

299. J. Fei, C. Lu, Adaptive fractional order sliding mode controller with neural estimator. J. Frankl. Inst. 355(5), 2369-2391 (2018). https://doi.org/10.1016/j. jfranklin.2018.01.006

300. H. Bao, J.H. Park, J. Cao, Non-fragile state estimation for fractional-order delayed memristive BAM neural networks. Neural Netw. 119, 190-199 (2019). https:// doi.org/10.1016/j.neunet.2019.08.003

301. R. Li, X. Gao, J. Cao, Quasi-state estimation and quasi-synchronization control of quaternion-valued fractional-order fuzzy memristive neural networks: vector ordering approach. Appl. Math. Comput. 362(2018), 124572 (2019). https://doi.org/10.1016/j. amc.2019.124572

302. B. Hu, Q. Song, Z. Zhao, Robust state estimation for fractional-order complex-valued delayed neural networks with interval parameter uncertainties: LMI approach. Appl. Math. Comput. 373, 125033 (2020). https://doi.org/10.1016/j.amc.2020.125033

303. M. Hui, C. Wei, J. Zhang, H. Ho-Ching Iu, N. Luo, R. Yao, L. Bai, Finite-time projective synchronization of fractional-order memristive neural networks with mixed time-varying delays. Complexity 2020, 1-16 (2020)
304. W. Li, X. Gao, R. Li, Dissipativity and synchronization control of fractional-order memristive neural networks with reaction-diffusion terms. Math. Methods Appl. Sci. 42(18), 7494-7505 (2019)

305. D. Sierociuk, I. Petráš, Modeling of heat transfer process by using discrete fractional-order neural networks, in 2011 16th International Conference on Methods and Models in Automation and Robotics, MMAR 2011. IEEE, pp. 146-150 (2011)

306. Y. Zhang, Y. Sun, P. Phillips, G. Liu, X. Zhou, S. Wang, A multilayer perceptron based smart pathological brain detection system by fractional Fourier entropy. J. Med. Syst. (2016). https://doi.org/10.1007/ s10916-016-0525-2

307. E.N. Mobarez, A. Sarhan, M. Ashry, Fractional order PID based on a single artificial neural network algorithm for fixed wing uavs, in ICENCO 2019-2019 15th International Computer Engineering Conference: Utilizing Machine Intelligence for a Better World, (1), pp. 168-174 (2019)

308. X.Z. Liu, Z.T. Li, K.N. Wu, Boundary Mittag-Leffler stabilization of fractional reaction-diffusion cellular neural networks. Neural Netw. 132, 269-280 (2020). https://doi.org/10.1016/j.neunet.2020.09.009

309. K. Ding, Q. Zhu, Impulsive method to reliable sampleddata control for uncertain fractional-order memristive neural networks with stochastic sensor faults and its applications. Nonlinear Dyn. 100(3), 2595-2608 (2020). https://doi.org/10.1007/s11071-020-05670-y

310. L. Sánchez, J. Otero, D. Anseán, I. Couso, Health assessment of LFP automotive batteries using a fractional-order neural network. Neurocomputing $\mathbf{3 9 1}$, 345-354 (2020)

311. M. Roohi, C. Zhang, Y. Chen, Adaptive model-free synchronization of different fractional-order neural networks with an application in cryptography. Nonlinear Dyn. 100(4), 3979-4001 (2020). https://doi.org/ 10.1007/s11071-020-05719-y

312. M. Blasik, Numerical method for the one phase $1 \mathrm{D}$ fractional Stefan problem supported by an artificial neural network. (2), 1-20. (2019). arXiv:1909.13638 